\title{
Effects of Strength Training Using Unstable Surfaces on Strength, Power and Balance Performance Across the Lifespan: A Systematic Review and Meta-analysis
}

\author{
David G. Behm ${ }^{1} \cdot$ Thomas Muehlbauer $^{2} \cdot$ Armin Kibele $^{3} \cdot$ Urs Granacher $^{2}$
}

Published online: 10 September 2015

(c) The Author(s) 2015. This article is published with open access at Springerlink.com

\begin{abstract}
Background The effectiveness of strength training on unstable surfaces (STU) versus stable surfaces (STS) or a control condition (CON; i.e. no training or regular training only) for strength, power and balance performance across the lifespan has not yet been investigated in a systematic review and meta-analysis.

Objective The aims of this systematic review and metaanalysis were to determine the general effects of STU versus STS or CON on muscle strength, power and balance in healthy individuals across the lifespan and to investigate whether performance changes following STU are age specific.

Data Sources A computerized systematic literature search was performed in the electronic databases PubMed and Web of Science from January 1984 up to February 2015.

Study Eligibility Criteria Initially, 209 articles were identified for review. Only controlled trials were included if they investigated STU in healthy individuals and tested at least one measure of maximal strength, strength
\end{abstract}

This article is part of the Topical Collection on Exercise to improve mobility in healthy aging.

Urs Granacher

urs.granacher@uni-potsdam.de

1 School of Human Kinetics and Recreation, Memorial University of Newfoundland, St. John's, NL, Canada

2 Division of Training and Movement Sciences, Research Focus Cognition Sciences, University of Potsdam, Am Neuen Palais 10, Building 12, 14469 Potsdam, Germany

3 Institute for Sports and Sport Science, University of Kassel, Kassel, Germany endurance, muscle power, or static/dynamic balance. In total, 22 studies met the inclusion criteria.

Study Appraisal and Synthesis Methods The included studies were coded for the following criteria: age, sex, training status, training modality, exercise and test modality. Effect size measures included within-subject standardized mean differences $\left(\mathrm{SMD}_{\mathrm{w}}\right)$ and weighted between-subject standardized mean differences $\left(\mathrm{SMD}_{\mathrm{b}}\right)$. Heterogeneity between studies was assessed using $I^{2}$ and $\chi^{2}$ statistics. The methodological quality of each study was assessed using the Physiotherapy Evidence Database (PEDro) Scale.

Results Our search failed to identify studies that examined the effects of STU versus STS or CON in children and middle-aged adults. However, four studies were identified that investigated the effects of STU versus CON or STS in adolescents, 15 studies were identified in young adults and three studies were identified in old adults. Compared with CON, STU produced medium effects on maximal strength in young adults and no effects to medium effects in old adults. In addition, large effects were detected on strength endurance in adolescents and in young adults; in old adults, a small effect was found. With regard to muscle power, medium effects were observed in young adults and small effects were observed in old adults. Further, large effects were found for static and dynamic balance in old adults, but only a small effect was found for dynamic balance in young adults. The comparison of STU and STS revealed inconsistent results as indicated by training-induced changes in favour of STU, as well as STS. Small to medium effects were found for maximal strength in adolescents in favour of STS, and small effects were found in young adults in favour of STU. With regard to strength endurance, large effects were found in adolescents in favour of STS and small effects were found in favour of STU. 
Additionally, we detected small effects in young adults in favour of STU. In terms of muscle power, no effects were observed in adolescents but medium effects were found in favour of STS in young adults. With regard to balance, small effects were detected in adolescents for static and dynamic balance in favour of STU. In young adults, small effects were found for static balance in favour of STS. With regard to dynamic balance, the analysis revealed small effects in young adults in favour of STU.

Limitations The quality of the included studies was rather low, with mean PEDro scores of 5.8, 4.0 and 5.0 for studies including adolescents, young adults and old adults, respectively. Further, trivial to considerable heterogeneity between studies (i.e. $0 \% \leq I^{2} \leq 96 \%$ ) was detected.

Conclusions Compared with CON, STU is effective in improving muscle strength, power and balance in adolescents, young adults and old adults. However, inconsistent results were particularly found in adolescents and young adults when the specific effects of STU were compared with those of STS. We conclude that the performance of STU compared with STS has limited extra effects on muscle strength, power and balance performance in healthy adolescents and young adults. Given that our systematic search did not identify studies that examined the effects of STU versus STS in children, middle-aged adults and old adults, further research of high methodological quality is needed to determine whether there are additive effects of STU as compared with STS in those age groups.

\section{Key Points}

This systematic review and meta-analysis determined the effects of strength training on unstable surfaces (STU) versus stable surfaces (STS) or a control condition (CON) on measures of muscle strength, power and balance, administered in the form of controlled trials in healthy individuals across the lifespan (aged $\geq 6$ years).

Our analyses revealed that STU, as compared with $\mathrm{CON}$, is an effective means to improve measures of muscle strength in healthy adolescents, young adults and old adults, and to improve variables of power and balance in young and old adults.

In adolescents and young adults, the specific comparison of STU with STS resulted in contradictory findings, and thus the use of unstable as compared with stable surfaces during strength training is not recommended in healthy adolescents and young adults if the goal is to enhance performance on stable surfaces.

\section{Introduction}

Devices with varying degrees of instability are frequently employed for athletic and everyday performance enhancement, balance promotion and musculoskeletal health to mimic the demands of the various tasks in applied settings $[1,2]$. There are many devices that attempt to provide an unstable surface. These devices include airpressurized balls (e.g. Swiss, physio or exercise balls), hemispherical balls with an inflated dome side and a hard rubber flat side (e.g. the BOSU $^{\circledR}$ ball), inflatable discs, wobble or balance boards, foam tubes, and high- and lowdensity foam platforms, as well as many other related devices. Unstable devices promote postural disequilibrium or imbalance, as postural sway may project the centre of mass beyond the device's area of support. Unstable devices also promote postural disequilibrium as the surface distorts (e.g. a low-density foam cushion) readily in response to the reaction forces associated with changes in the centre of pressure.

According to the principle of training specificity [3, 4], training must simulate as closely as possible the demands of the task or activity. Tasks such as sports and fitness activities, occupational tasks and activities of daily living often occur on relatively unstable surfaces (e.g. skiing, snowboarding, skating, walking and working in icy or muddy conditions). Willardson [5] stated that "the optimal method to promote increases in balance, proprioception and spinal stability for any given sport is to practice the skill itself on the same surface on which the skill is performed in competition". Similarly, Schmidtbleicher [6] stated that intermuscular coordination can only be developed by practising the movement for which coordination is sought. Unfortunately, with some seasonal sports, specific training is not possible year round (e.g. skiing in the summer or baseball in the snow). Therefore, alternative challenges using unstable surfaces could be included in training to provide a progressive overload and to stimulate strength and balance adaptations. Strength training on stable surfaces (STS), such as squats, deadlifts and Olympic lifts, is conducted with a moderate degree of instability [1, 7-10]. Greater degrees of instability are provided when strength training is conducted on unstable surfaces (STU) or with unstable implements.

Proponents of unstable devices suggest that the greater instability may stress the neuromuscular system to a greater extent than STS $[11,12]$. The rationale is that destabilizing training environments may enhance neuromuscular adaptations and training specificity, while providing a more varied and effective training stimulus. The Canadian Society for Exercise Physiology position stand [7] indicates that there are functional health benefits of STU 
(e.g. improved joint stability and reduced lower-extremity injury rates). In addition, improved strength, balance and functional performance have been reported following STU in primarily young healthy adults [13]. Further, STU appears to be a suitable training regimen to be implemented in the rehabilitative context and/or the geriatric context [8, 9]. In fact, the application of STU is not restricted to young healthy adults. For example, more 'vulnerable' cohorts due to biological aging (i.e. seniors) or maturational processes (i.e. children, adolescents) may particularly benefit from STU because surface instability allows and demands lower training loads but at the same time sufficiently and adequately stimulates the neuromuscular system of youth and seniors [1, 2, 7-9]. Given that only a few studies have investigated the effects of STU as a promising training regimen in seniors, more research is needed to elucidate the effects of STU in seniors and to find out whether it is more effective than traditional STS. Further, the biological concept of 'critical or sensitive maturational periods', i.e. periods during which ontogenetic development reaches a qualitatively new level that provides opportunities for further improvement of an organ, tissue and/or physiological function [14], may imply that the adaptive potential following STU is also high in youth. In fact, Behm and Colado Sanchez [2] propagated the use of STU for performance enhancements in youth. However, it is not known whether increases in muscle strength, power and balance performances are comparable across the lifespan. A lifespan approach appears to be important because experts have reported in narrative reviews that STU is a meaningful and promising training regimen for youth, adults and seniors [2, 15]. However, these statements lack verification, which is why there is a need for this meta-analysis across the age continuum. Further, meta-analyses represent the highest evidence level on the evidence pyramid [16]. Given that this topic has been addressed by narrative reviews only [2, 15], a metaanalysis may further our knowledge in this area by providing in-depth information (representing a high level of evidence).

Therefore, a synthesis of the literature is needed to determine whether or not STU provides additional effects on measures of muscle strength, power and balance in comparison with STS. The purpose of the present systematic review and meta-analysis was to provide a study on the highest evidence level in evidence-based medicine regarding the effects of STU on measures of muscle strength, power and balance administered in the form of controlled trials in healthy individuals across the lifespan. It is hypothesized that STU produces similar or even larger performance enhancements than STS because the performance of STU is highly demanding for the neuromuscular system (i.e. additional joint and postural stability are needed during exercise). Further, on the basis of expert opinion [2, 15] and selected studies [17, 18], we expected that STU would be particularly suitable and effective in seniors and youth because it has previously been shown that strength training using low loads produced similar or even larger performance gains in these age groups.

\section{Methods}

We followed the Preferred Reporting Items for Systematic Reviews and Meta-Analysis (PRISMA) statement guidelines provided by Moher et al. [19] when conducting our systematic review and meta-analysis.

\subsection{Literature Search}

We performed a computerized systematic literature search in PubMed and Web of Knowledge from January 1984 up to February 2015 to capture all relevant articles that investigated the effectiveness of STU versus STS. The following Boolean search strategy was applied using the operators 'AND', 'OR' and 'NOT': (( 'instability resistance training' OR 'instability strength training' OR 'free-weight training' OR 'instability weight-bearing exercise program' OR 'instability weight-bearing strengthening program' OR 'instability weight-lifting exercise program' OR 'weightlifting strengthening program') AND (balance OR 'balance performance' OR posture OR 'postural balance') AND ('strength' OR 'muscle strength' OR 'muscular strength' OR power OR 'muscle power' OR 'muscular power') NOT ('natural surfaces' OR 'unilateral exercises')). The search was limited to the English language, the human species and full-text availability of original articles reporting a controlled trial in an academic journal. Further, we checked the reference lists of each included article, and we analysed relevant review articles $[1,2,5,7-9,20,21]$ in an effort to identify additional suitable studies for inclusion in the database.

\subsection{Selection Criteria}

To be eligible for inclusion, studies had to meet the following criteria: (1) the participants in the experimental groups had to be healthy subjects; (2) the participants had to be aged $\geq 6$ years; and ( 3 ) at least one strength, power and/ or balance performance outcome had to be reported in the study. Studies were excluded if (1) they did not have a controlled study design; (2) they included patients or people with diseases; or (3) it was not possible to extract means and standard deviations from the results section (i.e. text, tables or graphs) or the authors did not respond to our inquiries. On the basis of the defined inclusion and exclusion criteria, two 
independent reviewers (TM, UG) screened potentially relevant papers by analysing the titles, abstracts and full texts of the respective articles to elucidate their eligibility.

\subsection{Coding of Studies}

Each study was coded for the following variables: number of participants, sex, age, training status (i.e. trained or untrained subjects), type of sport practised and experimental groups [i.e. STU, STS or a control condition $(\mathrm{CON}$; i.e. no training or regular training only)]. We coded interventions according to the applied training modalities, i.e. the training period (number of training weeks), training frequency (number of training sessions/week), training volume (number of sets/repetitions/duration per exercise, duration of a single training session) and training intensity [e.g. percentage of one-repetition maximum (1RM)]. If exercise progression was realized over the training period, the range of sets, repetitions or durations per exercise/session was stated. If training modalities were not reported in detail, the authors were contacted and missing information was requested. Our analyses focused on muscle strength, power and balance outcomes. Muscle strength findings were considered in terms of the following categories: maximal strength (e.g. 1RM), strength endurance (e.g. number of sit-ups) and power (e.g. jump height). Balance was classified as either static (e.g. time during a one-legged stance) or dynamic (e.g. timed walking distance). For studies that reported multiple parameters within one of these outcome categories, the most representative parameter was included in our analysis. In terms of muscle strength, 1RM was defined as the most important variable representing maximal strength. With regard to strength endurance, the number of sit-ups was used, and for muscle power, the countermovement jump (CMJ) height was applied. Concerning balance, the time during a one-legged stance was used as a proxy for static balance and the timed walking distance was used as a measure of dynamic balance. If the included studies did not report the results (i.e. means and standard deviations) of preand post-testing, we contacted the authors of those studies. In three cases [13, 22, 23], the authors responded and provided the relevant data. If the authors did not respond, the respective studies [24, 25] were excluded.

\subsection{Assessment of Methodological Quality and Statistical Analyses}

The methodological quality of all eligible intervention studies was assessed using the Physiotherapy Evidence Database (PEDro) Scale. The PEDro Scale rates internal study validity and the presence of statistical replicable information on a scale from 0 to 10 , with $\geq 6$ representing a cut-off score for high-quality studies [26]. The predetermined cut-off score of $\geq 6$ points was not a criterion for studies to be included or excluded. Two independent reviewers (UG, TM) performed quality assessments of the included studies. When any disagreement between the raters occurred, a consensus meeting was held and an additional rating was obtained from a third assessor (DGB) to achieve a consensus.

To verify the effectiveness of STU and STS for measures of muscle strength, power and balance, we computed withinsubject standardized mean differences as $\mathrm{SMD}_{\mathrm{w}}=$ (mean pre-test value - mean post-test value)/standard deviation pre-test value, and between-subject standardized mean differences as $\mathrm{SMD}_{\mathrm{b}}=$ (mean post-test value in intervention group - mean post-test value in control group)/pooled variance [27]. In addition, the standardized mean difference was adjusted for the respective sample size according to the following formula: $g=\left(1-\frac{3}{4 N_{i}-9}\right)$, with $N_{i}$ representing the total sample size [27, 28]. Furthermore, the included studies were weighted with respect to the magnitude of the respective standard error, using Review Manager version 5.3.4 (The Nordic Cochrane Centre, The Cochrane Collaboration; Copenhagen; 2008). A random-effects metaanalysis model was applied to compute the overall standardized mean difference in Review Manager version 5.3.4. As a function of the respective outcome measure (e.g. 1RM, timed walking distance), $\mathrm{SMD}_{\mathrm{w}}$ and $\mathrm{SMD}_{\mathrm{b}}$ could be negative or positive. Thus, a positive $\mathrm{SMD}_{\mathrm{w}}$ value would indicate performance improvements (i.e. an increase in jump height) from pre- to post-intervention within one experimental group, and a negative $\mathrm{SMD}_{\mathrm{w}}$ value would indicate a performance decrement (i.e. a decrease in jump height) within one experimental group. In terms of $\mathrm{SMD}_{\mathrm{b}}$, a positive value would be indicative of a performance change in favour of STU, whereas a negative value would be indicative of a performance change in favour of STS or CON. According to Cohen [29], values for $\mathrm{SMD}_{\mathrm{w}} / \mathrm{SMD}_{\mathrm{b}}$ of $0.00 \leq 0.49$ indicate small effects, values of $0.50 \leq 0.79$ medium effects and values of $>0.80$ large effects. Heterogeneity between studies was assessed using $I^{2}$ and $\chi^{2}$ statistics. On the basis of the recommendations from Deeks et al. [30], values of $0 \% \leq I^{2} \leq 40 \%$ would indicate trivial heterogeneity, values of $30 \% \leq I^{2} \leq 60 \%$ moderate heterogeneity, values of $50 \% \leq I^{2} \leq 90 \%$ substantial heterogeneity and values of $75 \% \leq I^{2} \leq 100 \%$ considerable heterogeneity.

\section{Results}

\subsection{Study Characteristics}

Figure 1 displays a flow chart summarizing our systematic search, which identified a total of 209 controlled trials. 


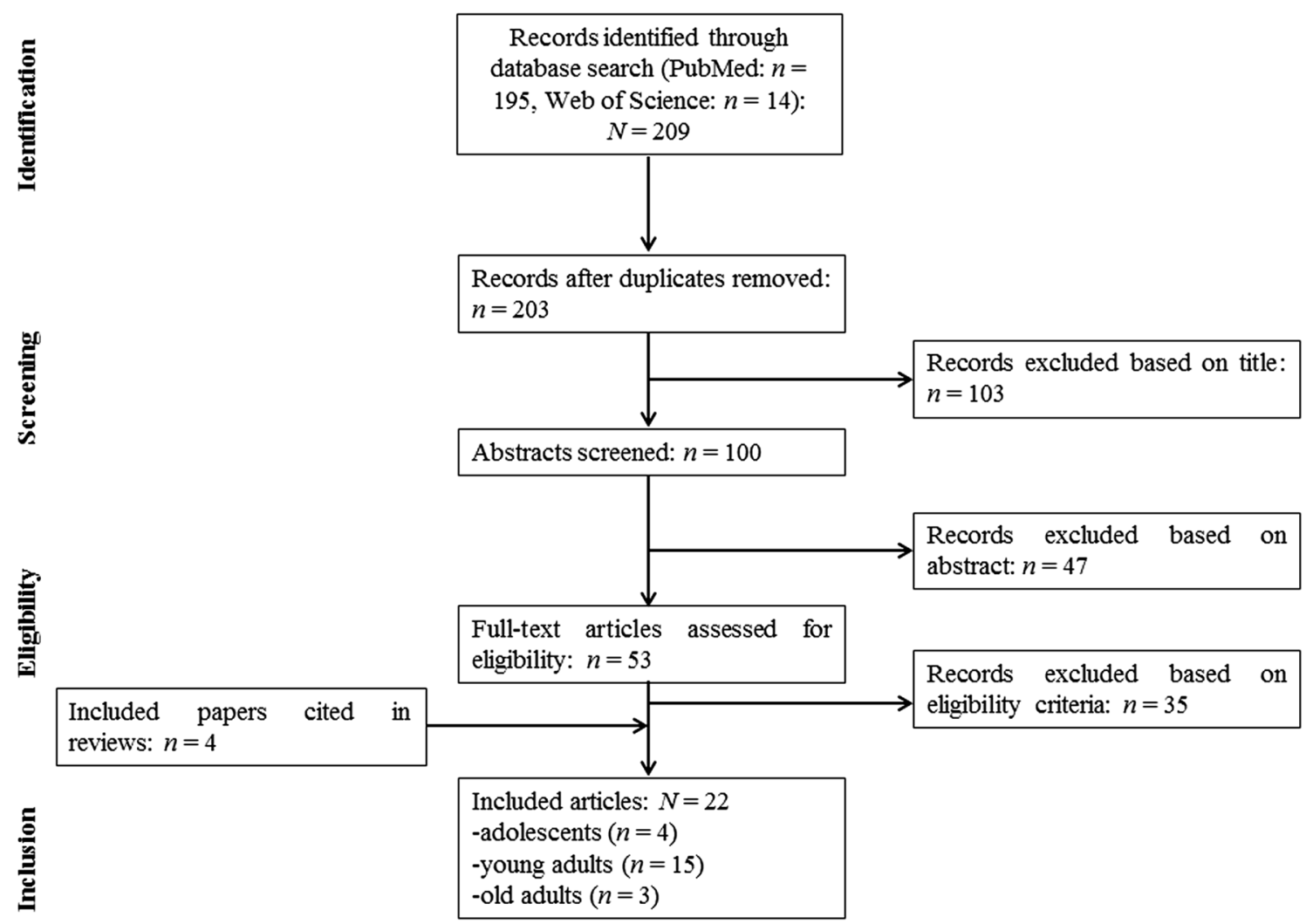

Fig. 1 Flowchart illustrating the different phases of the search and study selection

After removal of duplicates and exclusion of ineligible articles, 18 studies remained. We identified four additional articles [31-34] from the reference lists of the included papers and from already published review articles $[1,2,5$, $7-9,20,21]$. Therefore, 22 studies were included in the final analysis. Our lifespan approach was limited to adolescents (four studies), young adults (15 studies) and old adults (three studies) because our systematic search did not reveal any studies investigating the effects of STU on muscle strength, power and balance performance in children and middle-aged adults. Table 1 displays the main characteristics of the 22 included studies.

Four studies $(1 \times$ STU versus CON, $3 \times$ STU versus STS) examined the effects of STU in adolescents [35-38]. Three of them were conducted with trained subjects [35, 36,38 ] and one with untrained subjects [37]. A total of 106 adolescents participated in the four studies, and 52 of those received STU. The sample size of the STU groups ranged from eight to 18 subjects. STU protocols that were conducted in adolescents involved core strength training (e.g. supine leg bridge, side bridge) and plyometric training on stable surfaces (e.g. CMJ, drop jump [DJ]) or unstable surfaces (e.g. balance pad, Dynair ${ }^{\circledR}$ Cushion, Swiss ball). To evaluate the effects of STU in adolescents, one study [36] used a test for assessment of maximal isometric muscle strength (MIMS) (i.e. MIMS trunk flexors/extensors), two studies [35, 37] used tests for assessment of strength endurance (i.e. Bourban trunk muscle strength test, prone stabilization core stability test) and three studies [36-38] used tests for assessment of muscle power [i.e. CMJ, DJ, standing long jump (SLJ), Multiple 5 Bounds test]. Furthermore, two studies [37, 38] tested for static balance (e.g. one-legged stance time), as well as dynamic balance [i.e. reach distance in the Star Excursion Balance test (SEBT), $Y$ balance test]. The training period for STU ranged from 6 to 9 weeks, with a total of 12-27 training sessions. The numbers of sets and repetitions per exercise ranged from 2 to 5 and from 5 to 25 , respectively. The duration per exercise lasted between 15 and $50 \mathrm{~s}$. Lastly, the duration of a single training session ranged from 25 to 35 minutes. None of the studies provided specific information on (perceived) training intensity (e.g. the Borg Scale). General information on progression during STU was given in terms of an increase in the number of sets, repetitions or duration per exercise. Additionally, training progression was achieved by increasing the difficulty level of the respective exercises (i.e. modulation of the lever length or type of muscle action; increase of drop, jump or hurdle height; reduction of base of support; addition of opposite limb movements). 


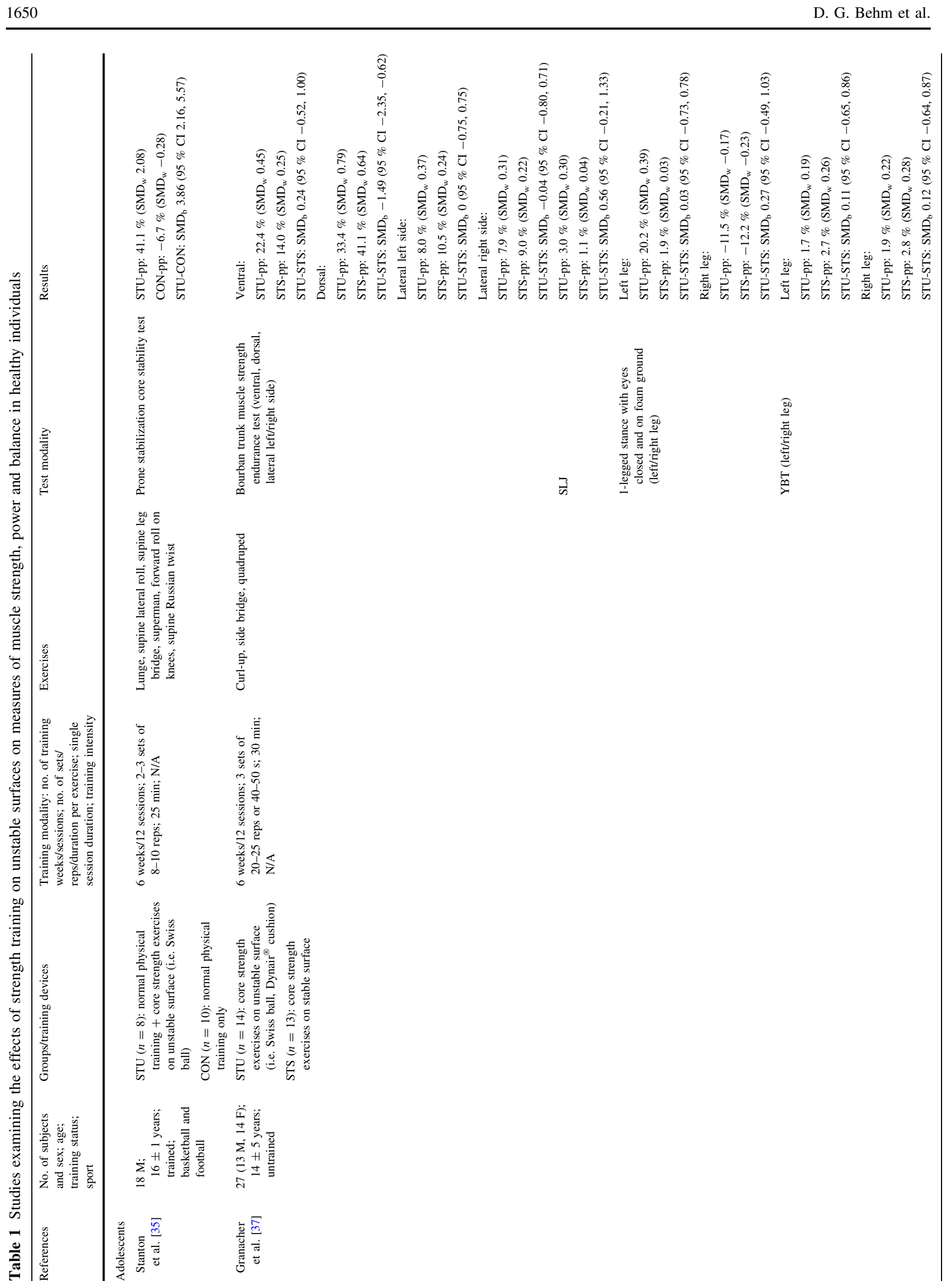

望 Springer 


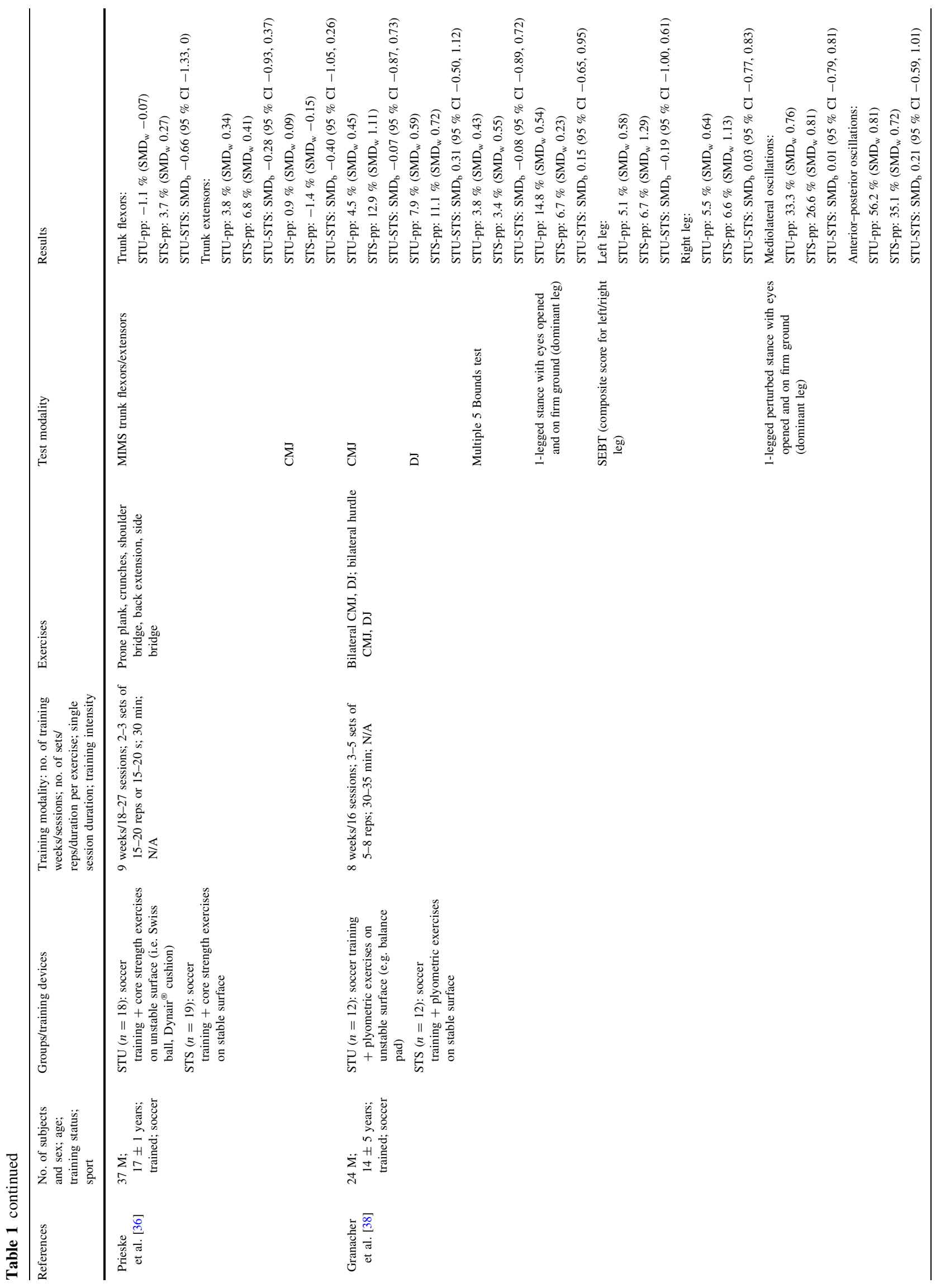




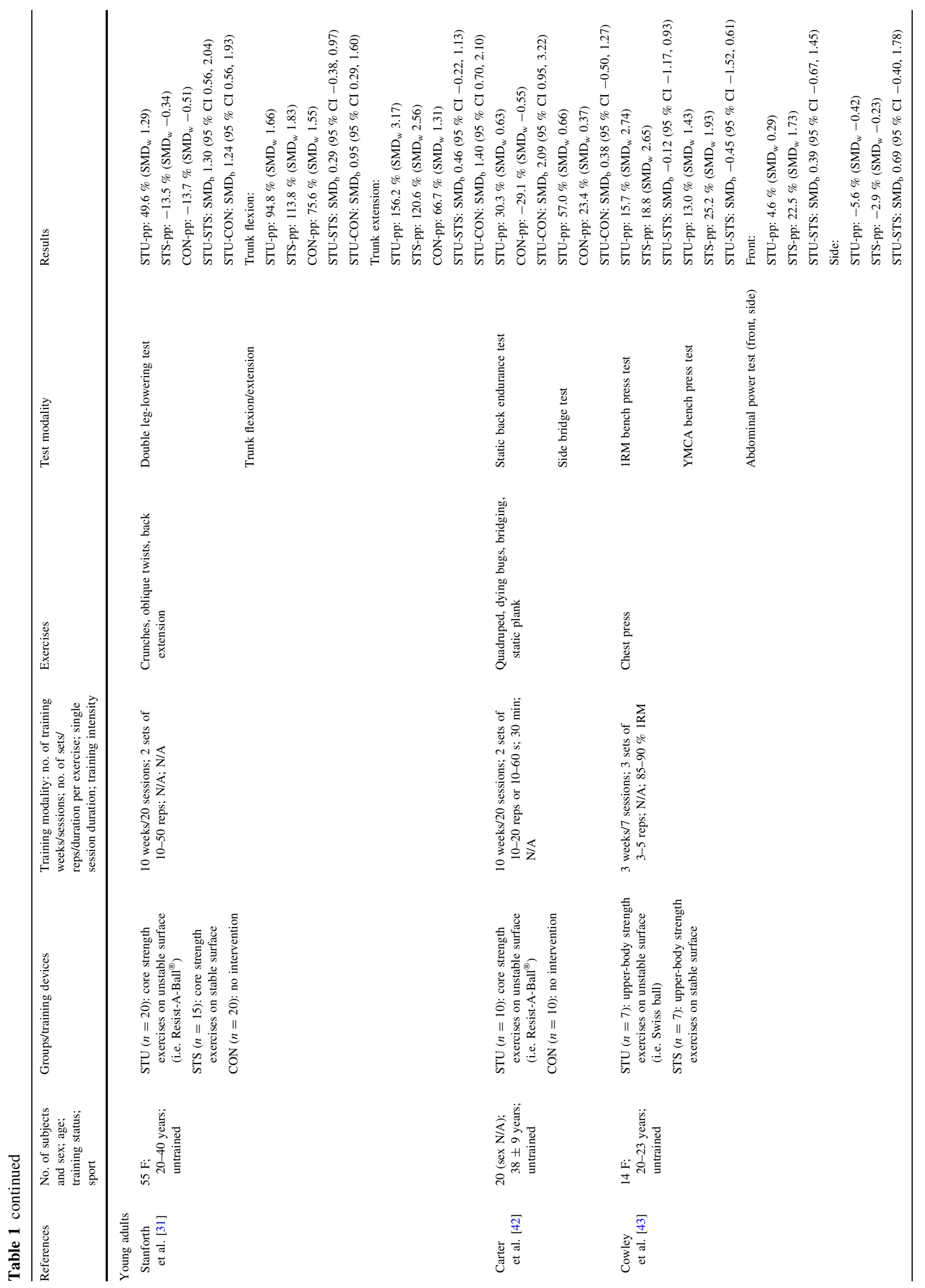




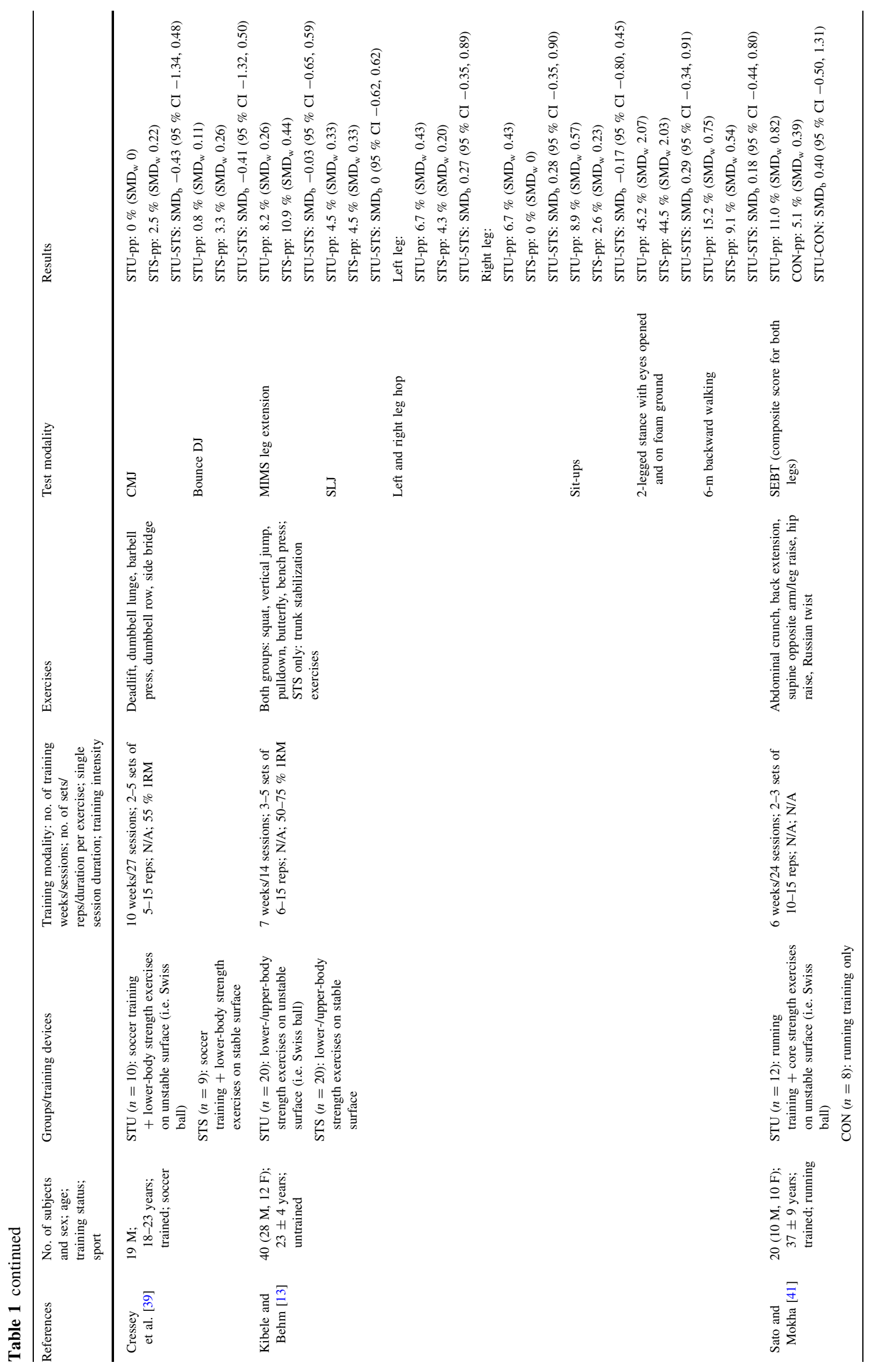




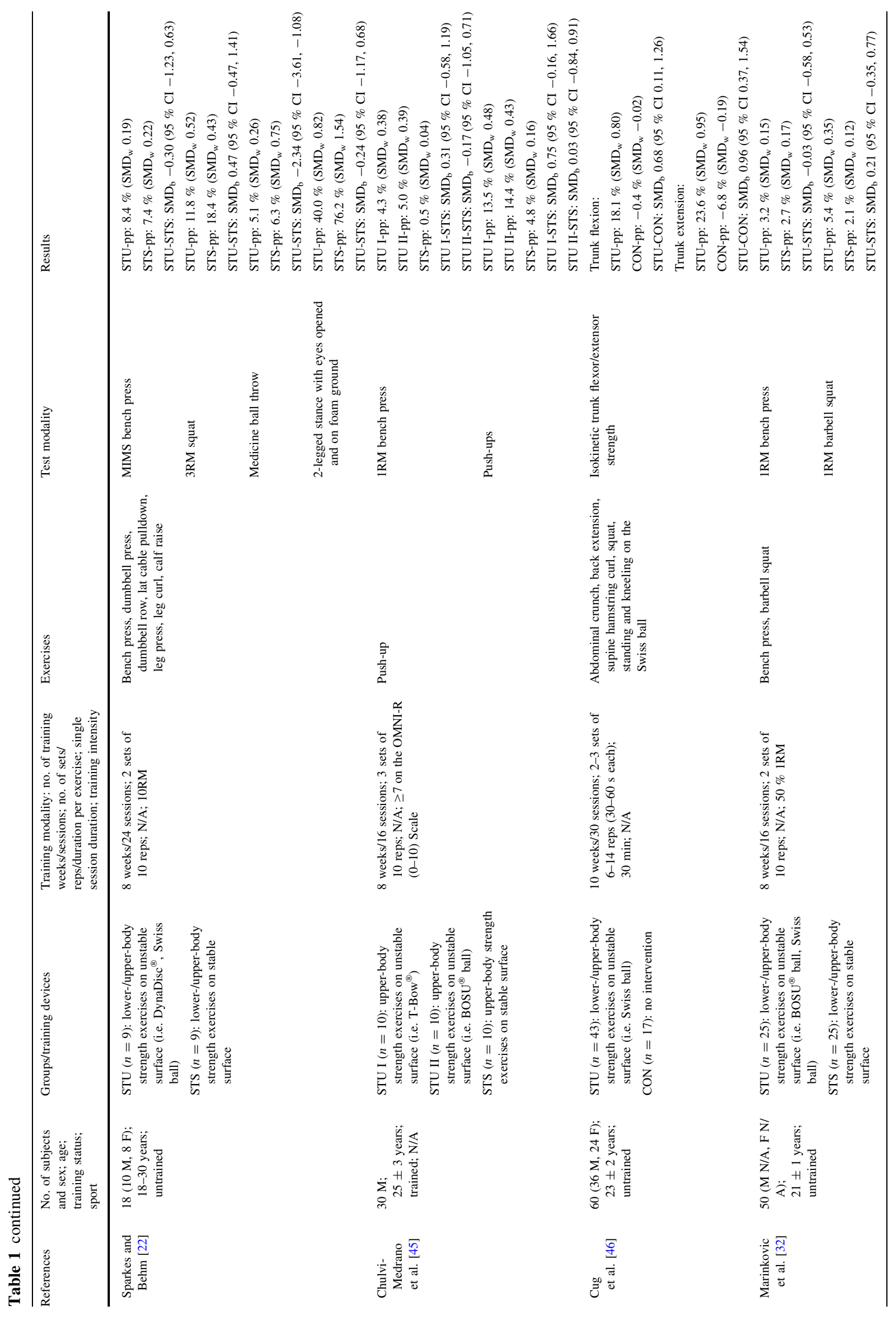




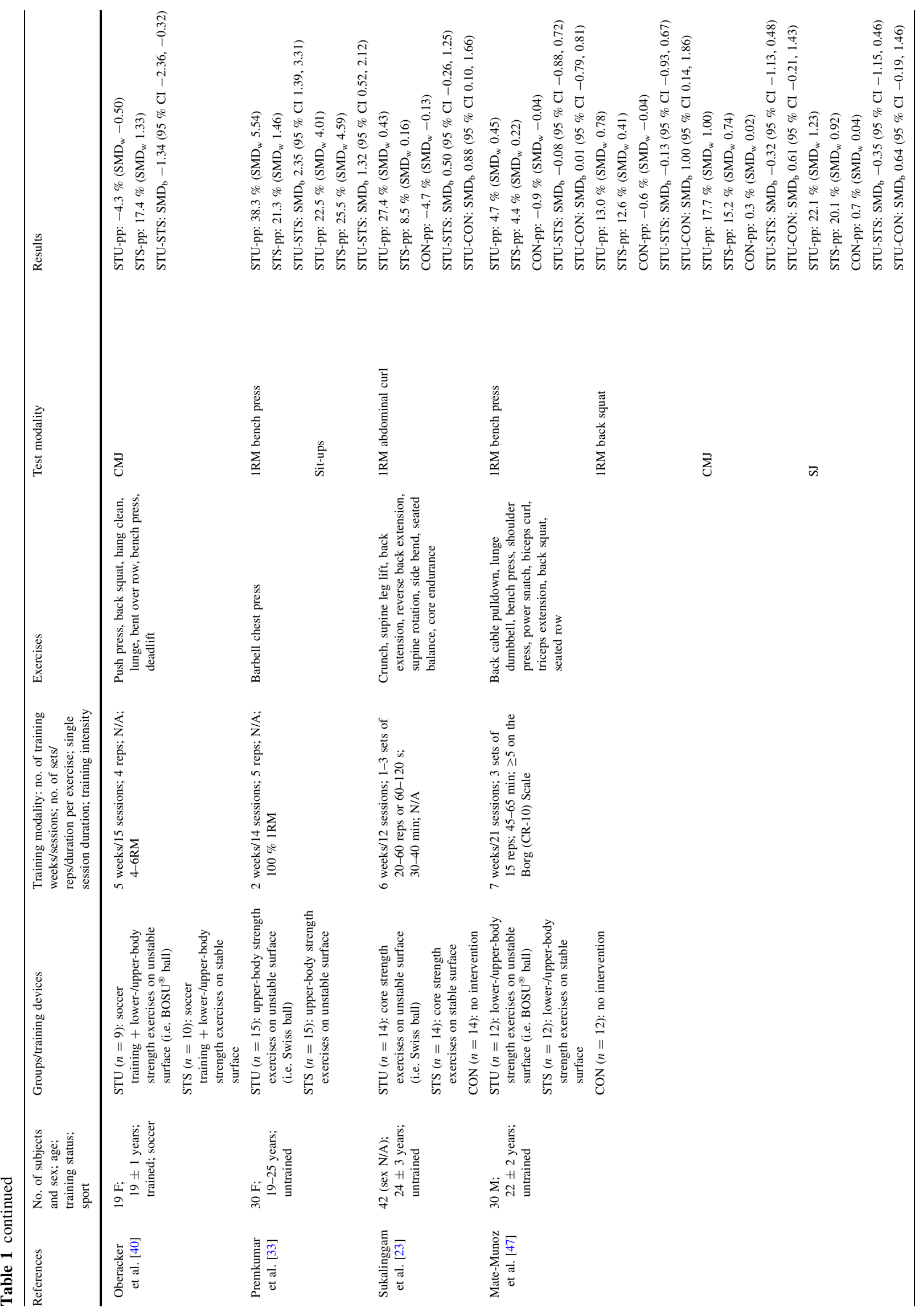




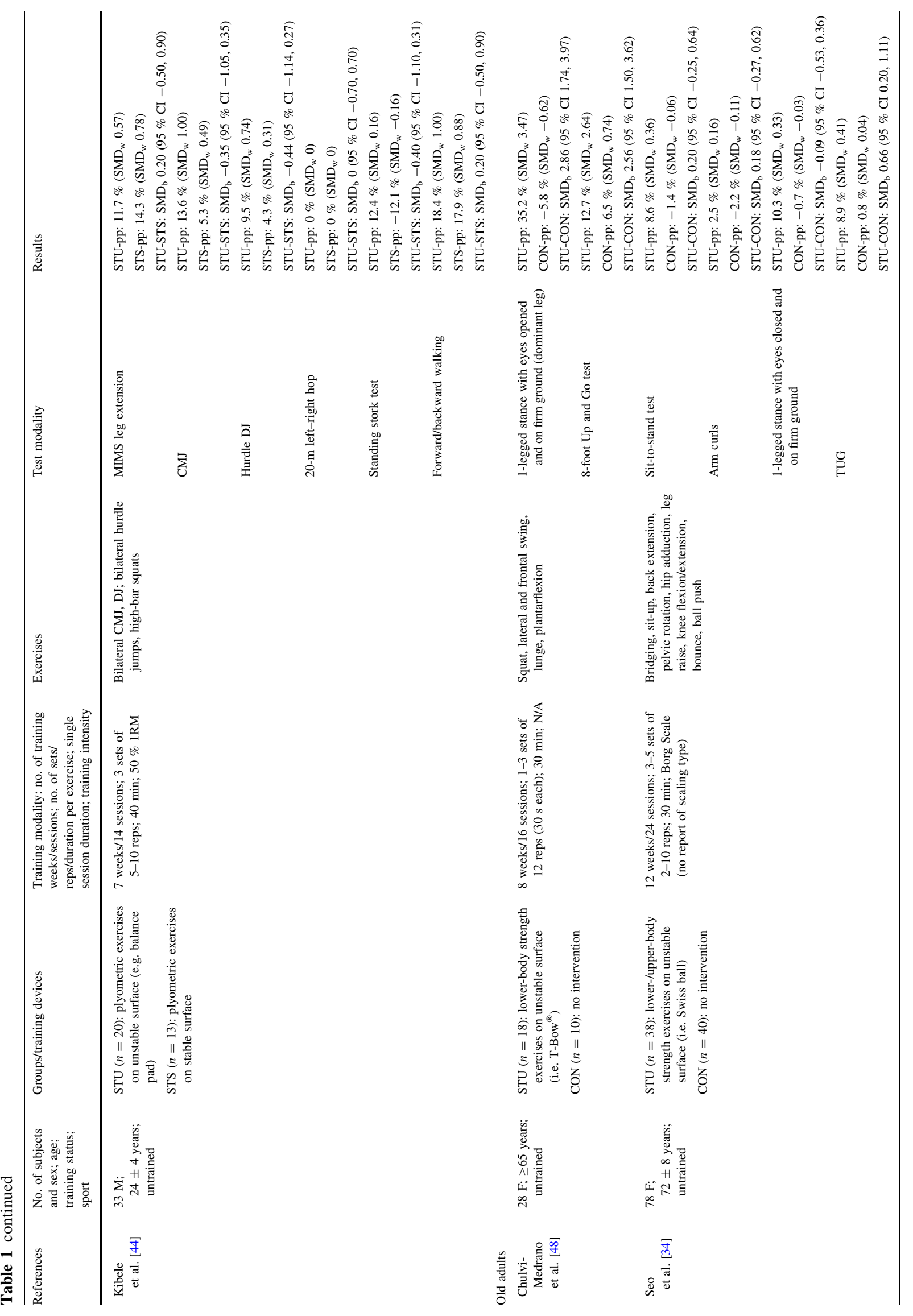




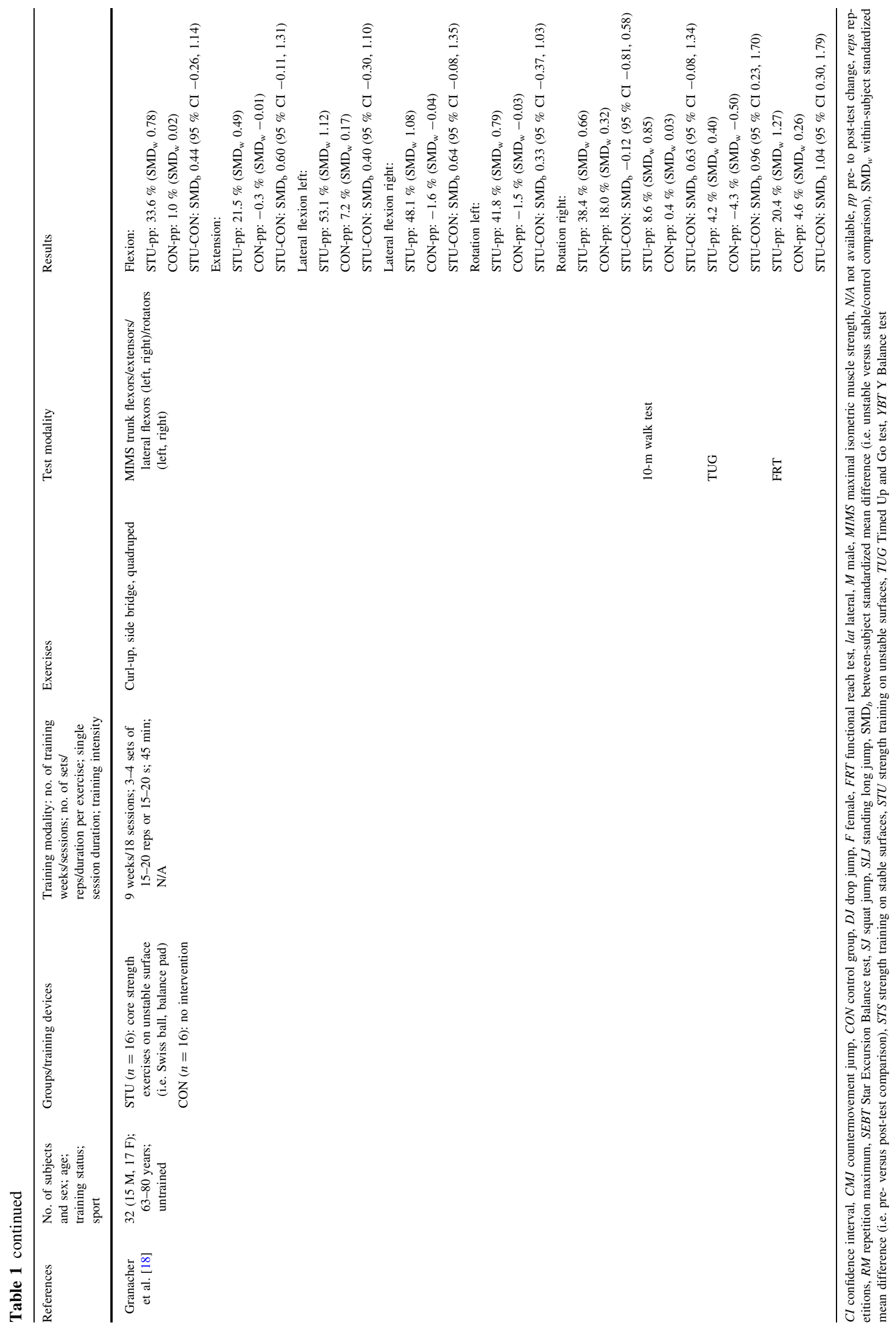


Further, our systematic search revealed 15 studies $(6 \times$ STU versus CON, $12 \times$ STU versus STS $)$ that investigated the effects of STU on measures of muscle strength, power and balance in young adults. Three of them included trained participants [39-41] and 12 included untrained participants [13, 22, 23, 31-33, 42-47]. A total of 480 young adults participated in the 15 studies, and 246 of those received STU. The sample size of the STU groups ranged from 7 to 43 subjects. STU protocols that were conducted in young adults involved core strength training (e.g. bridging, static plank) and lower-body strength exercises (e.g. dead lift, leg press), as well as upper-body strength exercises (e.g. chest press, dumbbell row), on unstable surfaces (e.g. a balance pad, BOSU ${ }^{\circledR}$ ball, DynaDisc $^{\circledR}$, Resist-A-Ball ${ }^{\circledR}$, Swiss ball, T-Bow ${ }^{\circledR}$ ). To evaluate STU effects in young adults, 11 studies [13, 22, 23, 31-33, 43-47] used a test for assessment of maximal muscle strength (i.e. 1RM abdominal curl, 1RM barbell/ back squat, 1RM bench press, double leg-lowering test, leg extension, YMCA bench press test), five studies [13, 31, 33, 42, 45] applied tests for assessment of muscular endurance (i.e. back extension, push-ups, side bridge test, sit-ups, static back endurance test, trunk flexion) and seven studies [13, 22, 39, 40, 43, 44, 47] used tests to determine muscle power (i.e. abdominal power test, CMJ, DJ, leg hop, medicine ball throw, SLJ). Furthermore, three studies $[13,22,44]$ tested for static balance (i.e. two-legged stance, standing stork test) and three studies [13, 41, 44] tested for dynamic balance (i.e. 6-m backward walking, forward/backward walking, SEBT). The training periods for STU in young adults ranged from 2 to 10 weeks, with a total of 7-30 training sessions. The numbers of sets and repetitions per exercise ranged from 1 to 5 and from 3 to 60 , respectively. The duration of single exercises lasted between 10 and $120 \mathrm{~s}$, and the duration of a single training session lasted between 30 and $65 \mathrm{~min}$. Information on (perceived) training intensity during STU in young adults was provided in nine studies and ranged from 50 to $100 \%$ $1 \mathrm{RM}, \geq 5$ on the Borg Scale (CR-10) and $\geq 7$ on the OMNI$\mathrm{R}$ Scale (0-10). General information on progression during STU was given in terms of an increase in the number of sets, the number of repetitions or the duration per exercise. Additionally, the difficulty level of STU exercises progressed over the course of the study by applying additional loads and by increasing drop, jump and hurdle height $[40$, 44].

Three studies $(3 \times$ STU versus CON $)$ examined the effects of STU in old adults [18, 34, 48]. All of them included untrained subjects. A total of 128 seniors participated in the three studies, and 72 of those received STU. The sample sizes of the STU groups ranged from 16 to 38 subjects. STU protocols in old adults involved core strength training (e.g. quadruped, side bridge) and lower- body strength exercises (e.g. squat, lunge), as well as upper-body strength exercises (e.g. back extension, sit-up), on unstable surfaces (e.g. a balance pad, Swiss ball, T-Bow $\left.{ }^{\circledR}\right)$. To evaluate the effects of STU in old adults, one study [18] used a test to determine MIMS (i.e. MIMS trunk flexors/extensors/lateral flexors/rotators) and another study [34] applied tests for assessment of muscular power (i.e. sit-to-stand test, arm curls). Furthermore, two studies $[34,48]$ tested for static balance (i.e. one-legged stance) and three studies $[18,34,48]$ tested for dynamic balance (i.e. 10-m walk test, functional reach test, Timed Up and Go test, 8-Foot Up and Go test). STU training periods in old adults lasted between 8 and 12 weeks, with a total of 16-24 training sessions. The applied numbers of sets and repetitions per exercise ranged between one and five and between 2 and 20, respectively. A single exercise lasted between 15 and $20 \mathrm{~s}$, and the duration of a single training session ranged from 30 to $45 \mathrm{~min}$. Information on perceived training intensity during STU in old adults was provided in one study [34] using the Borg Scale ( $\geq 7$ ) (no report of scaling type). General information on progression during STU was given in terms of an increase in the numbers of sets, repetitions or duration per exercise. Additionally, training progression was achieved by increasing the difficulty level of the respective STU exercise (i.e. modulation of lever length, range of motion or movement velocity, reduction of base of support).

\subsection{Methodological Quality of the Included Trials}

In general, the quality of the included studies was rather low, with mean PEDro scores of 5.8, 4.0 and 5.0 for studies examining adolescents, young adults and old adults, respectively. The predetermined cut-off score of $\geq 6$ on the PEDro Scale was achieved by three out of four studies in adolescents [36-38], none out of 15 studies in young adults and two out of three studies in old adults $[18,48]$ (Table 2).

\subsection{Effectiveness of Strength Training on Unstable Surfaces Versus Control Condition}

One study in adolescents [35], six studies in young adults $[23,31,41,42,46,47]$, and three studies in old adults [18, $34,48]$, but no studies in children and middle-aged adults, examined the effects of STU compared with CON (i.e. no training or regular training only) on measures of strength, power and balance (Table 1). A general forest plot for measures of muscle strength and balance is presented in Fig. 2a, b. Our analyses revealed large effects of STU on muscle strength (mean $\mathrm{SMD}_{\mathrm{b}}=0.91, I^{2}=61 \%$, $\chi^{2}=10.36$, degrees of freedom $(d f)=4, p=0.03$; five studies [23, 31, 42, 46, 47]; Fig. 2a) and balance outcomes (mean $\mathrm{SMD}_{\mathrm{b}}=1.18, I^{2}=82 \%, \chi^{2}=11.07, \quad d f=2$, 


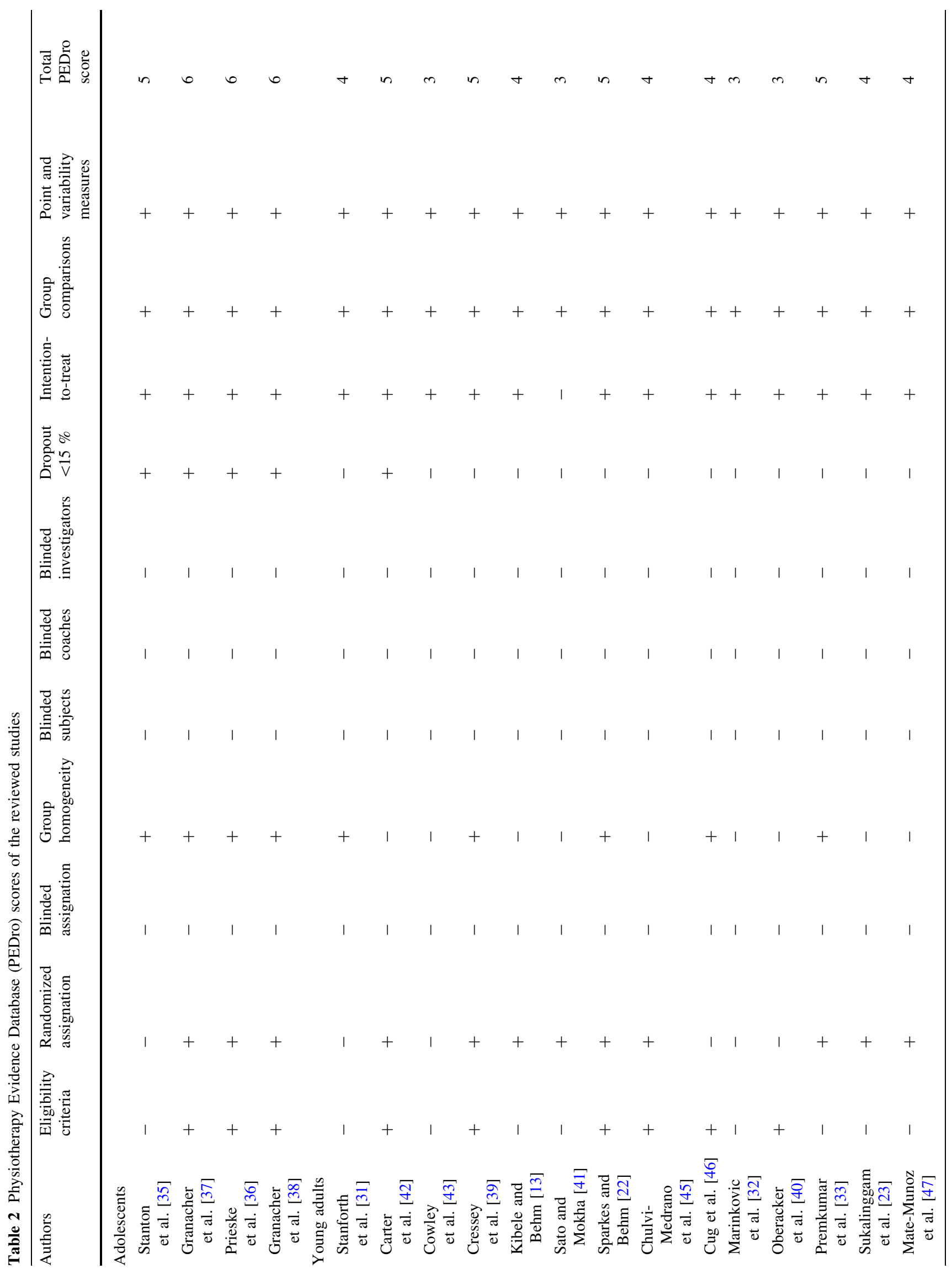




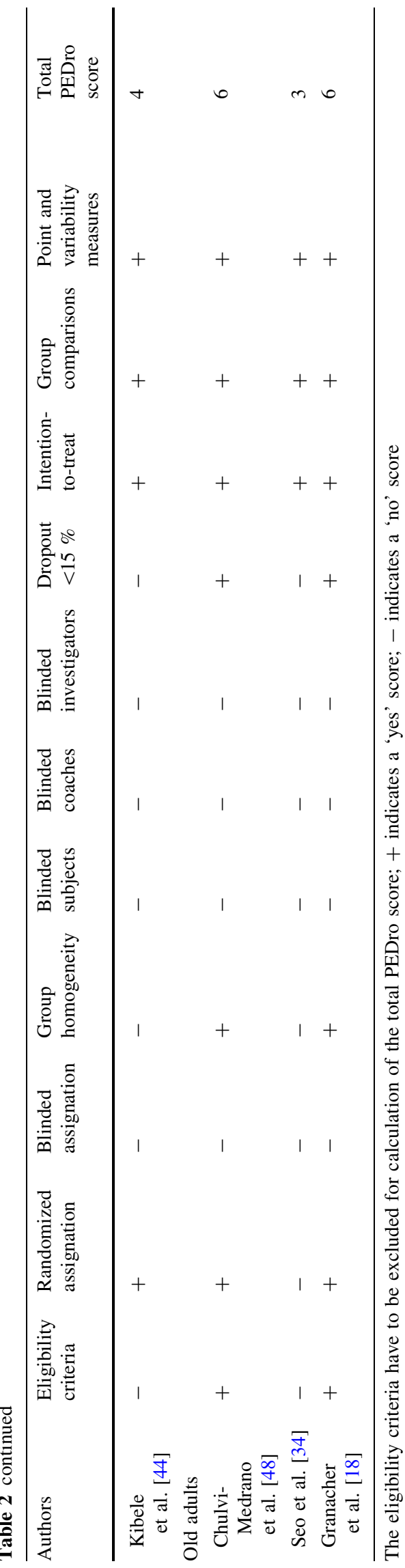

$p=0.004$; three studies $[18,34,48]$; Fig. 2b). Additionally, our item-specific analyses revealed that STU produced medium effects on variables of maximal strength in young adults (mean $\mathrm{SMD}_{\mathrm{b}}=0.72, I^{2}=44 \%, \quad \chi^{2}=5.38$, $d f=3, p=0.15$; four studies [23, 31, 46, 47]; Fig. 3a) and, depending on the analysed variable, no effects (i.e. maximal strength of the trunk rotators right) to medium effects [i.e. maximal strength of the trunk flexors, rotators, lateral flexors (left/right), lateral rotators (left)] in old adults $\left(\mathrm{SMD}_{\mathrm{b}}=-0.12\right.$ to 0.64 ; one study [18]). Additionally, our analysis revealed large effects of STU on measures of strength endurance in adolescents $\left(\mathrm{SMD}_{\mathrm{b}}=3.86\right.$; one study [35]) and in young adults (mean $\mathrm{SMD}_{\mathrm{b}}=1.42, I^{2}=65 \%, \chi^{2}=2.88, d f=1, p=0.09$; two studies [31, 42]; Fig. 3b). However, only a small effect was found in old adults $\left(\mathrm{SMD}_{\mathrm{b}}=0.18\right.$; one study [34]). Furthermore, medium effects on muscle power were detected for STU as compared with CON in young adults $\left(\mathrm{SMD}_{\mathrm{b}}=0.61-0.64\right.$; one study [47]) and a small effect in old adults $\left(\mathrm{SMD}_{\mathrm{b}}=0.20\right.$; one study [34]).

In terms of static balance, STU yielded large effects in old adults (mean $\mathrm{SMD}_{\mathrm{b}}=1.34, I^{2}=96 \%, \chi^{2}=23.03$, $d f=1, p<0.001$; two studies [34, 48]; Fig. 4a) in comparison with CON. Lastly, a small effect was detected for measures of dynamic balance in young adults $\left(\mathrm{SMD}_{\mathrm{b}}=0.40 ;\right.$ one study [41] $)$ and large effects in old adults $\left(\right.$ mean $\mathrm{SMD}_{\mathrm{b}}=1.18, \quad I^{2}=82 \%, \quad \chi^{2}=11.07$, $d f=2, p=0.004$; three studies [18, 34, 48]; Fig. 4b).

\subsection{Effectiveness of Strength Training on Unstable Versus Stable Surfaces}

Three studies in adolescents [36-38] and 12 studies in young adults [13, 22, 23, 31-33, 39, 40, 43-45, 47]—but no studies in children, middle-aged and old adults-examined the effects of STU compared with STS on measures of strength, power and balance (Table 1). A general forest plot for measures of muscle strength and balance is illustrated in Fig. 5a, b. Our analyses revealed small effects in favour of STU on muscle strength (mean $\mathrm{SMD}_{\mathrm{b}}=0.15$, $I^{2}=68 \%, \chi^{2}=46.92, d f=15, p<0.001 ; 15$ studies [13, 22, 23, 31-33, 36-40, 43-45, 47]; Fig. 5a) and balance (mean $\mathrm{SMD}_{\mathrm{b}}=0.09, \quad I^{2}=0 \%, \quad \chi^{2}=0.69, \quad d f=4$, $p=0.95$; five studies [13, 22, 37, 38, 44]; Fig. 5b). Additionally, our item-specific analyses revealed inconsistent results as indicated by training-induced changes in favour of STU (i.e. a positive $\mathrm{SMD}_{\mathrm{b}}$ value), as well as STS (i.e. a negative $S M D_{b}$ value). More specifically, small to medium effects were detected for measures of maximal strength in adolescents $\left(\mathrm{SMD}_{\mathrm{b}}=-0.66\right.$ to -0.28 ; one study [36]) in favour of STS, and small effects in young adults (mean $\mathrm{SMD}_{\mathrm{b}}=0.34, \quad I^{2}=68 \%, \chi^{2}=31.42$, $d f=10, p=0.0005 ; 10$ studies $[13,22,23,31-33,43-45$, 


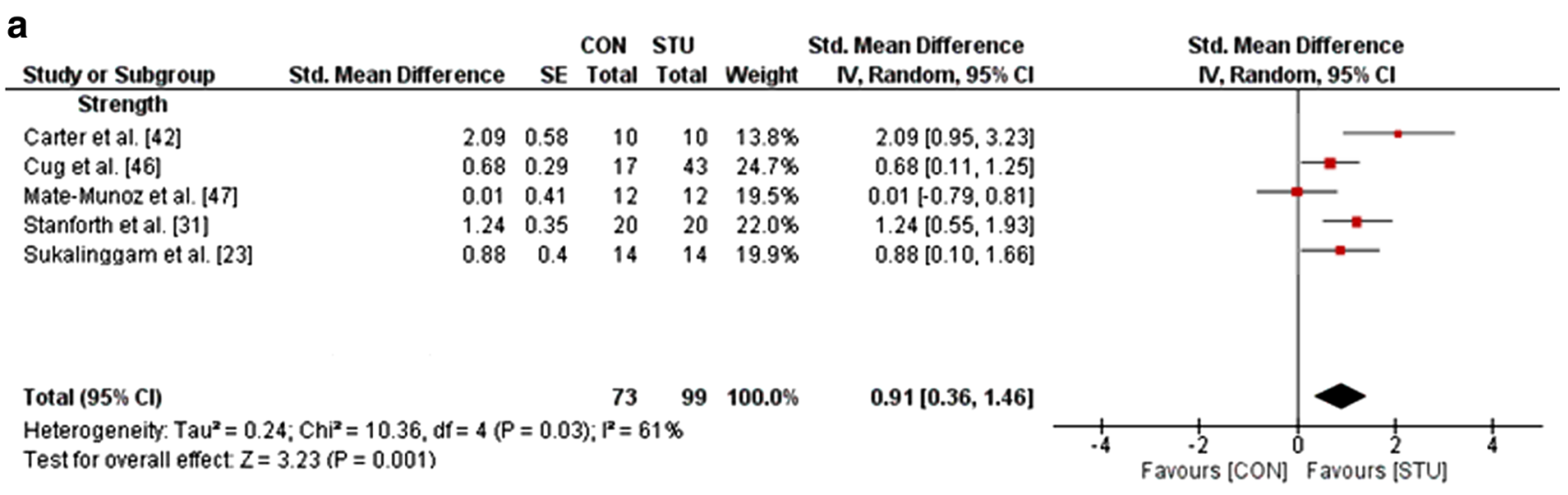

b

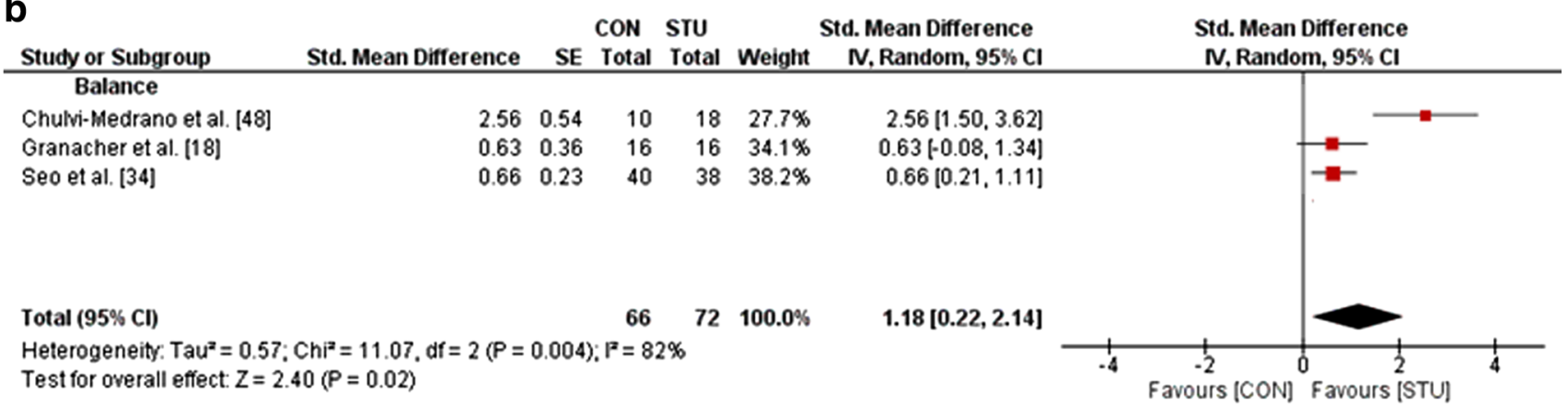

Fig. 2 Effects of strength training on unstable surfaces (STU) versus control condition (CON; i.e. no training or regular training only) on measures of strength (a) and balance (b). CI confidence interval, $d f$ degrees of freedom, $I V$ inverse variance, $S E$ standard error, Std. standard

47]; Fig. 6a) in favour of STU. In terms of strength endurance, large effects were observed in adolescents in favour of STS and small effects in favour of STU $\left(\mathrm{SMD}_{\mathrm{b}}=-1.49\right.$ to 0.24 ; one study [37]). In young adults, STU produced small effects on variables of strength endurance $\left(\right.$ mean $\mathrm{SMD}_{\mathrm{b}}=0.41, I^{2}=58 \%, \chi^{2}=9.49$, $d f=4, p=0.05$; four studies [13, 31, 33, 45]; Fig. 6b) in comparison with STS. In terms of muscle power, no effects were detected in adolescents (mean $\mathrm{SMD}_{\mathrm{b}}=0$, $I^{2}=44 \%, \chi^{2}=3.55, d f=2, p=0.17$; three studies [3638]; Fig. 7a); yet medium effects were observed in young adults in favour of STS (mean $\mathrm{SMD}_{\mathrm{b}}=-0.53, I^{2}=62 \%$, $\chi^{2}=15.93, d f=6, p=0.01$; seven studies $[13,22,39$, 40, 43, 44, 47]; Fig. 7b).

Furthermore, training-induced changes in balance performances following STU or STS were obtained, indicating small effects in adolescents for variables of static balance (mean $\mathrm{SMD}_{\mathrm{b}}=0.21, \quad I^{2}=0 \%, \quad \chi^{2}=0.04, \quad d f=1$, $p=0.83$; two studies [37, 38]; Fig. 8a) and dynamic balance $\left(\right.$ mean $\mathrm{SMD}_{\mathrm{b}}=0.08, I^{2}=0 \%, \chi \mathrm{i}^{2}=0.03, d f=1$, $p=0.87$; two studies [37, 38]; Fig. 9a) in favour of STU. In young adults, small effects were observed for measures of static balance in favour of STS (mean $\mathrm{SMD}_{\mathrm{b}}=-0.07$, $I^{2}=11 \%, \chi^{2}=2.23, d f=2, p=0.33$; three studies [13, 22, 44]; Fig. 8b) and for measures of dynamic balance in favour of STU (mean $\mathrm{SMD}_{\mathrm{b}}=0.19, I^{2}=0 \%, \chi^{2}=0$, $d f=1, p=0.97$; two studies [13, 44]; Fig. 9b).

\section{Discussion}

This is the first systematic literature review and metaanalysis to examine the effects of STU on measures of muscle strength, power and balance, administered in the form of controlled trials in healthy individuals across the lifespan. Twenty-two controlled trials (four in adolescents, 15 in young adults and three in old adults) were included in this review. The major findings of this review were that STU as compared with CON is effective in improving variables of muscle strength, power and balance in healthy adolescents, young adults and old adults when tested on stable surfaces. Further, small overall effects were found for measures of strength and balance in adolescents and young adults in favour of STU compared with STS. However, our item-specific analyses (e.g. maximal strength) revealed no consistent advantage of STU when it was compared with STS particularly in adolescents and young adults.

Adherents of STU would propose that such exercises provide advantages over STS, due to training specificity 


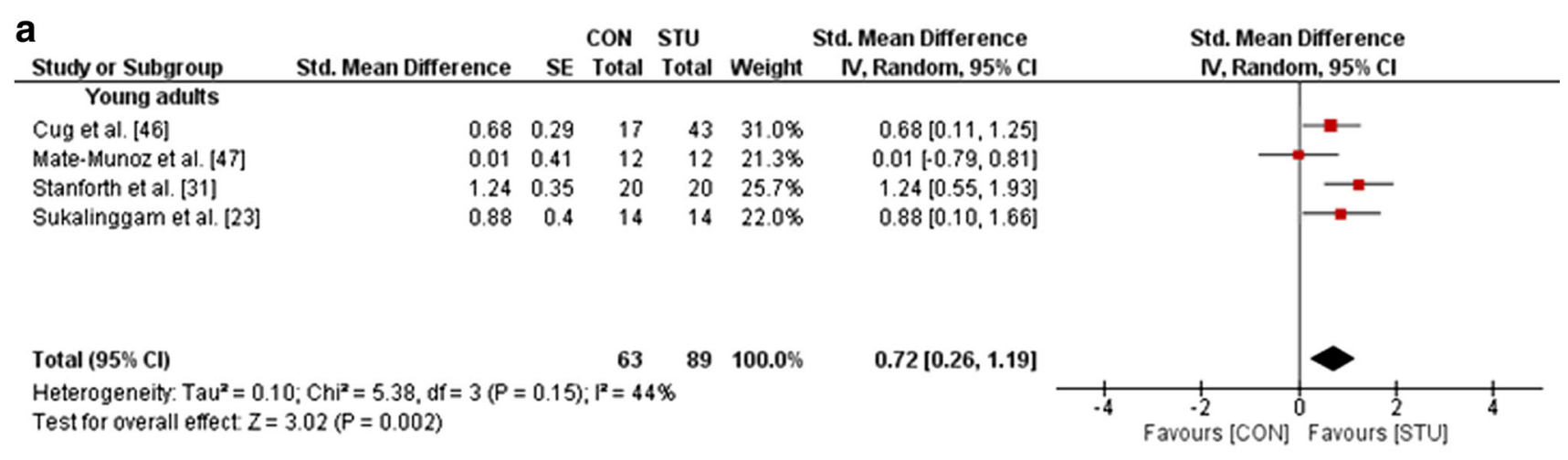

\begin{tabular}{|c|c|c|c|c|c|}
\hline Studvor Subaroun & Std Mean Difference & SF & CON & sru & Weiaht \\
\hline Young adults & & & & & \\
\hline Carter et al. [42] & 2.09 & 0.58 & 10 & 10 & $41.5 \%$ \\
\hline Stanforth et al. [31] & 0.95 & 0.34 & 20 & 20 & $58.5 \%$ \\
\hline Total $(95 \% \mathrm{Cl})$ & & & 30 & 30 & $100.0 \%$ \\
\hline
\end{tabular}

Fig. 3 Effects of strength training on unstable surfaces (STU) versus control condition (CON; i.e. no training or regular training only) on measures of maximal strength (a) and strength endurance (b) in
$1.42[0.32,2.52]$

Std. Mean Difference
N. Random, $95 \% \mathrm{Cl}$

$2.09[0.95 .3 .23]$

$0.95[0.28,1.62]$
Std. Mean Difference $\mathrm{N}$, Random, $95 \% \mathrm{Cl}$

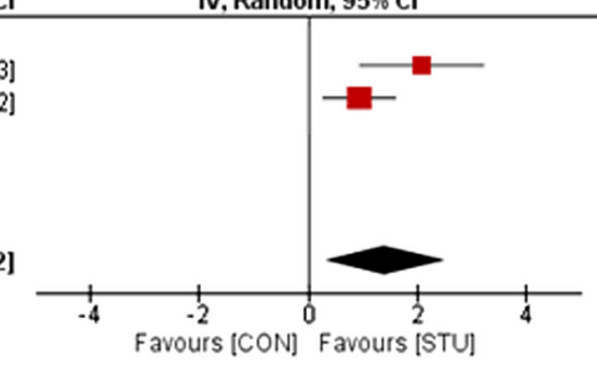

healthy young adults. $C I$ confidence interval, $d f$ degrees of freedom, $I V$ inverse variance, $S E$ standard error, $S t d$. standard

a

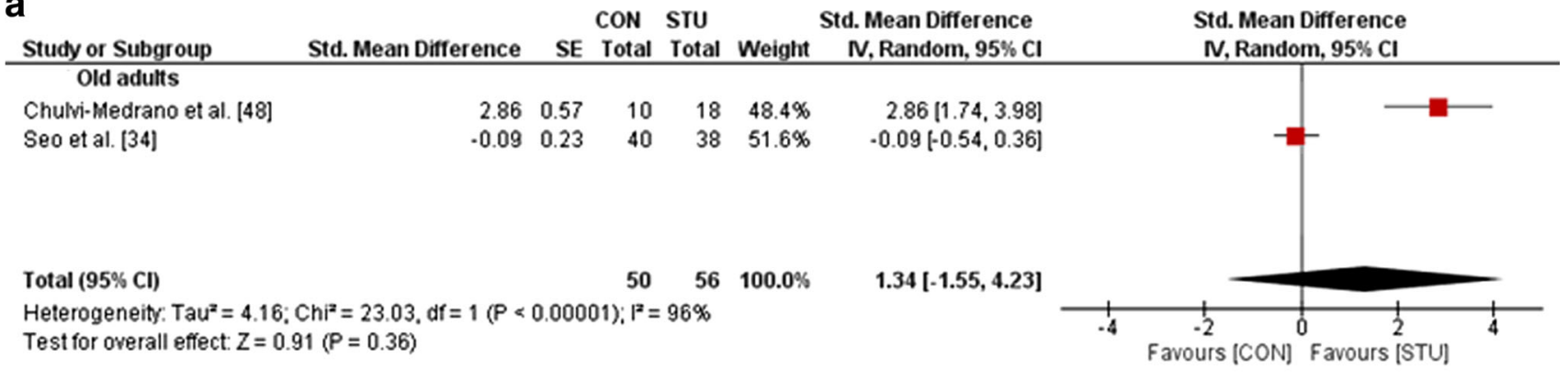

b

\begin{tabular}{|c|c|c|c|c|c|c|}
\hline Study or Subgroup & Std. Mean Difference & SE & $\begin{array}{l}\text { CON } \\
\text { Total }\end{array}$ & $\begin{array}{l}\text { STU } \\
\text { Total }\end{array}$ & Weight & $\begin{array}{l}\text { Std. Mean Difference } \\
\text { N. Random, } 95 \% \mathrm{Cl}\end{array}$ \\
\hline Old adults & & & & & & \\
\hline Chulvi-Medrano et al. [48] & 2.56 & 0.54 & 10 & 18 & $27.7 \%$ & $2.56[1.5$ \\
\hline Granacher et al. [18] & 0.63 & 0.36 & 16 & 16 & $34.1 \%$ & $0.63[-0.08,1.3$ \\
\hline Seo et al. [34] & 0.66 & 0.23 & 40 & 38 & $38.2 \%$ & $0.66[0.21,1.1$ \\
\hline
\end{tabular}

Total (95\% CI)

$\begin{array}{lll}66 & 72 \quad 100.0 \%\end{array}$

Heterogeneity: Tau $^{2}=0.57: \mathrm{Chi}^{2}=11.07 . \mathrm{df}=2(P=0.004): \mathrm{P}^{\mathrm{z}}=82 \%$

Test for overall effect: $Z=2.40(P=0.02)$

Fig. 4 Effects of strength training on unstable surfaces (STU) versus control condition (CON; i.e. no training or regular training only) on measures of static balance (a) and dynamic balance (b) in healthy old
$0.66[0.21,1,11]$

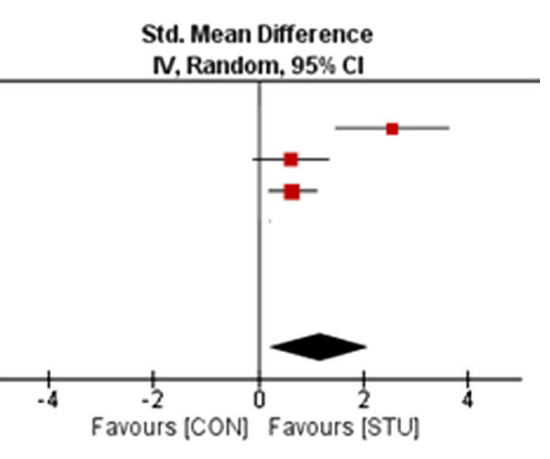

adults. $C I$ confidence interval, $d f$ degrees of freedom, $I V$ inverse variance, $S E$ standard error, $S t d$. standard 


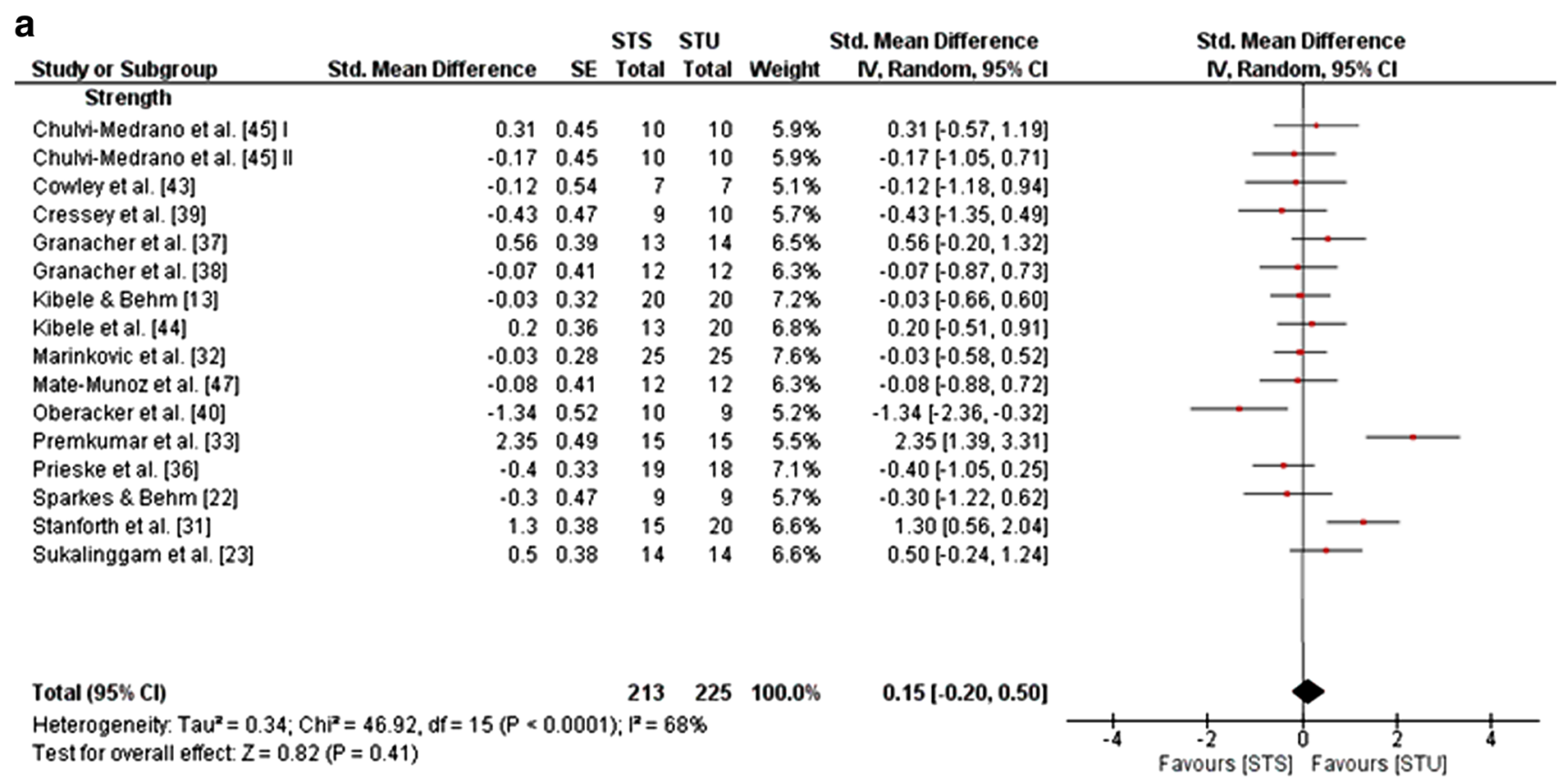

b

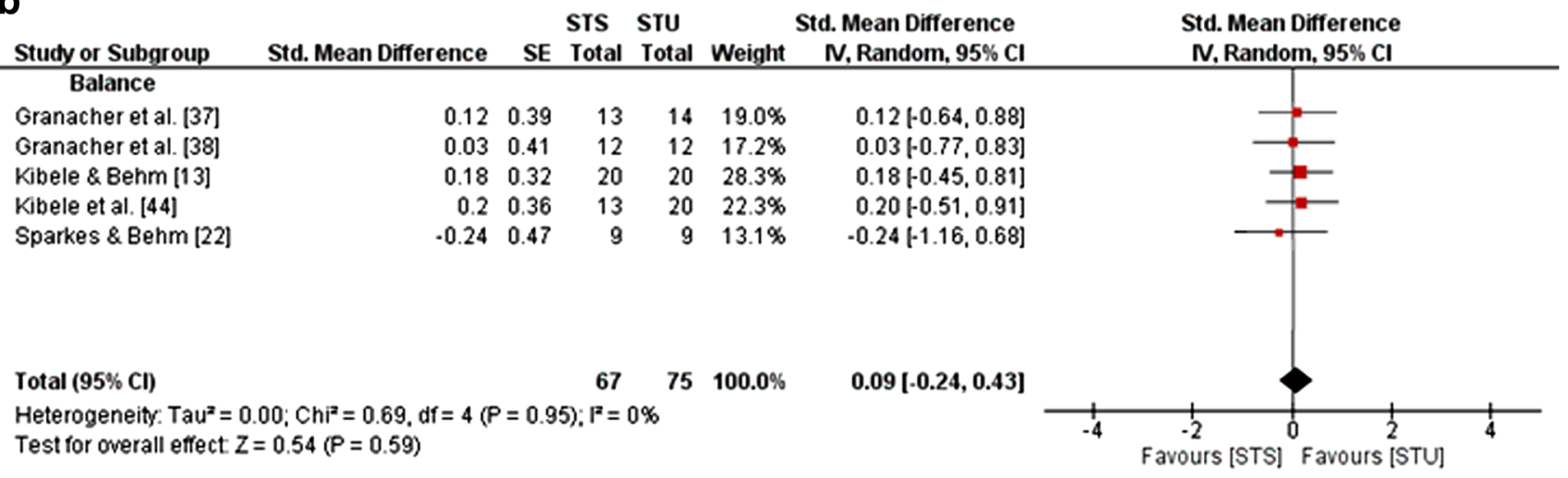

Fig. 5 Effects of strength training on unstable surfaces (STU) versus stable surfaces (STS) on measures of strength (a) and balance (b). $C I$ confidence interval, $d f$ degrees of freedom, $I V$ inverse variance, $S E$ standard error, $S t d$. standard

principles (i.e. exercising on unstable devices for unstable task demands during sports, fitness and in the workplace) $[3,4]$, while the greater degree of instability provides greater stress and thus greater opportunities for training adaptations of the neuromuscular and balance systems [11, 12]. The question of training specificity could not be directly addressed in this review, as the testing measures were typically laboratory based. The studies often used tests such as a one-legged stance, as well as strength and power measures, such as bench press, squat, CMJ, SJ and others, that were performed under relatively stable and stationary conditions (Table 1). The proposed training specificity of STU should be more apparent in tests performed under more unstable conditions (e.g. jumping from or landing on a balance pad). However, a counter-argument would concede that exercises used for STU are also typically static (stationary) exercises (performed without translational movement) and thus STU training specificity would not apply to dynamic, mobile, unstable activities, such as ice hockey, beach volleyball or soccer on a muddy field. For example, static STU [13] and dynamic STU [44] did not provide additional benefit on a dynamic balance test nor on a 20-m hopping test for speed. Balance improvements with the hopping test would have been expected to improve limb power output and decrease contact time, resulting in faster speeds. While Sparkes and Behm [22] found no significant training-specific differences following 8 weeks of static STU and STS, they did report a trend $(p=0.06)$ for the STU group to improve the stable to unstable chest press force ratio to a greater degree $(25 \%)$ 


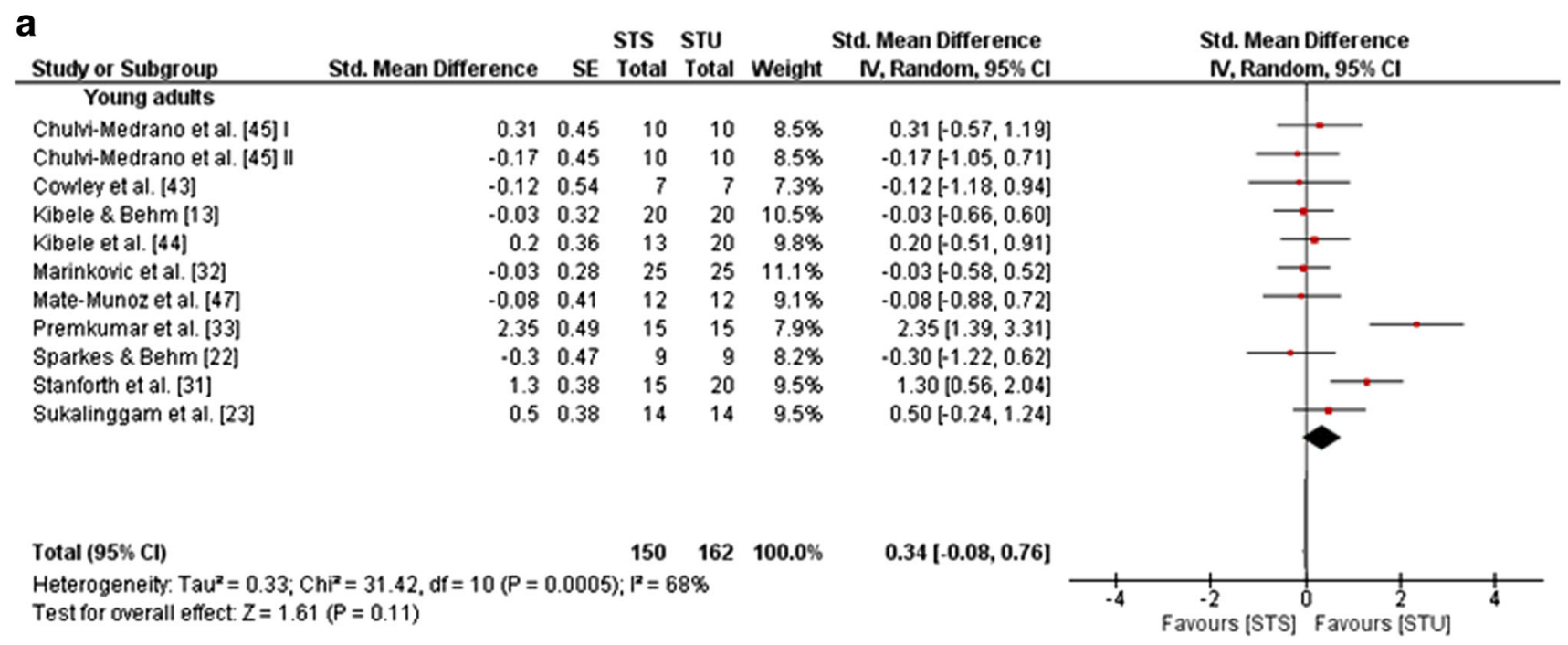

b

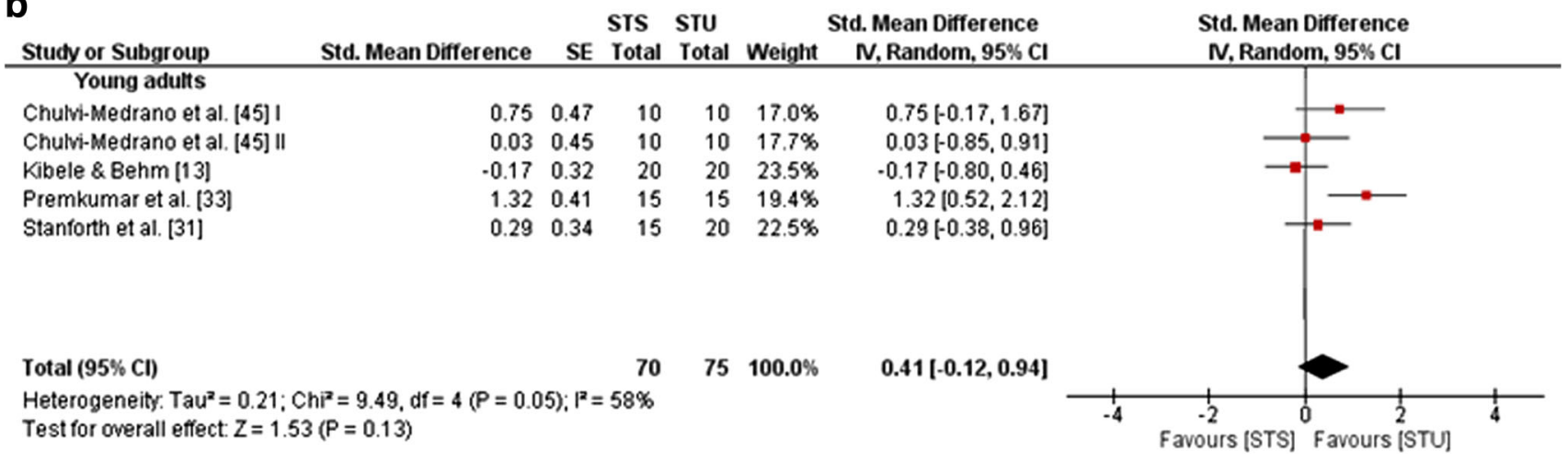

Fig. 6 Effects of strength training on unstable surfaces (STU) versus stable surfaces (STS) on measures of maximal strength (a) and strength endurance (b) in healthy young adults. $C I$ confidence interval, $d f$ degrees of freedom, $I V$ inverse variance, $S E$ standard error, $S t d$. standard

than the stable group $(11 \%)$. Although the trunk is supine upon a stable bench during the bench press, the load is suspended above the body, and the movement must be controlled with appropriate trunk and joint muscle stabilization. However, on the basis of the available data, it seems that STU does not provide systematic trainingspecific balance advantages over STS, irrespective of the age group considered (i.e. adolescent and young adults).

The lack of superiority of STU for balance measures may illustrate a dose-response or intensity-response relationship. While specific studies comparing the extent of centre of pressure excursions with STU versus STS are not available, it is generally accepted that STS involving freeweight lifts provides moderate levels of instability $[1,8,9]$. The placement and movement of bars, dumbbells and other implements on the shoulders (i.e. squats), above the body (i.e. shoulder presses, cleans, snatches) or in front of the body (i.e. bicep curls), for example, places disruptive torques outside the centre of gravity, challenging the system to maintain equilibrium. Although the challenges to postural stability may be greater during performance of a resistive exercise on an unstable surface, the present results demonstrate that this greater degree of stress does not lead to greater systematic balance improvements in adolescents and young adults.

The lower force and power outputs $[1,8,9,20,49$ 52] - as well as the decreased movement velocity and range of motion [50] - associated with STU could result in less rigorous strength and power training adaptations. However, not all studies that have investigated the effectiveness of STU reported force reductions under unstable conditions [43, 53, 54]. The Canadian Society for Exercise Physiology position stand [7] warns that "From a performance standpoint, unstable devices should not be utilized when hypertrophy, absolute strength, or power is the primary training goal, because force generation, power output, and movement velocity are impaired and may be insufficient to stimulate the desired adaptations, especially in 


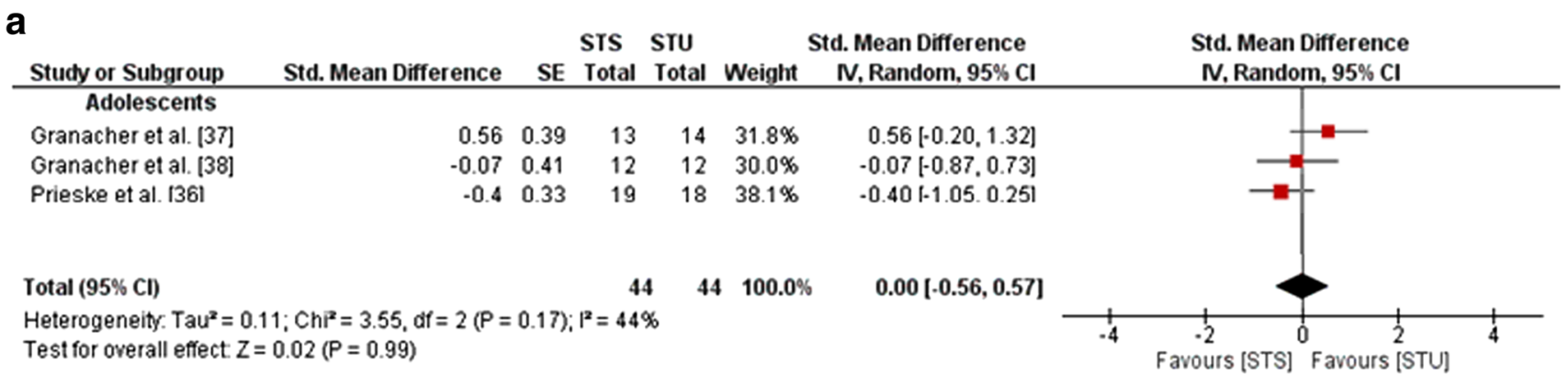

b

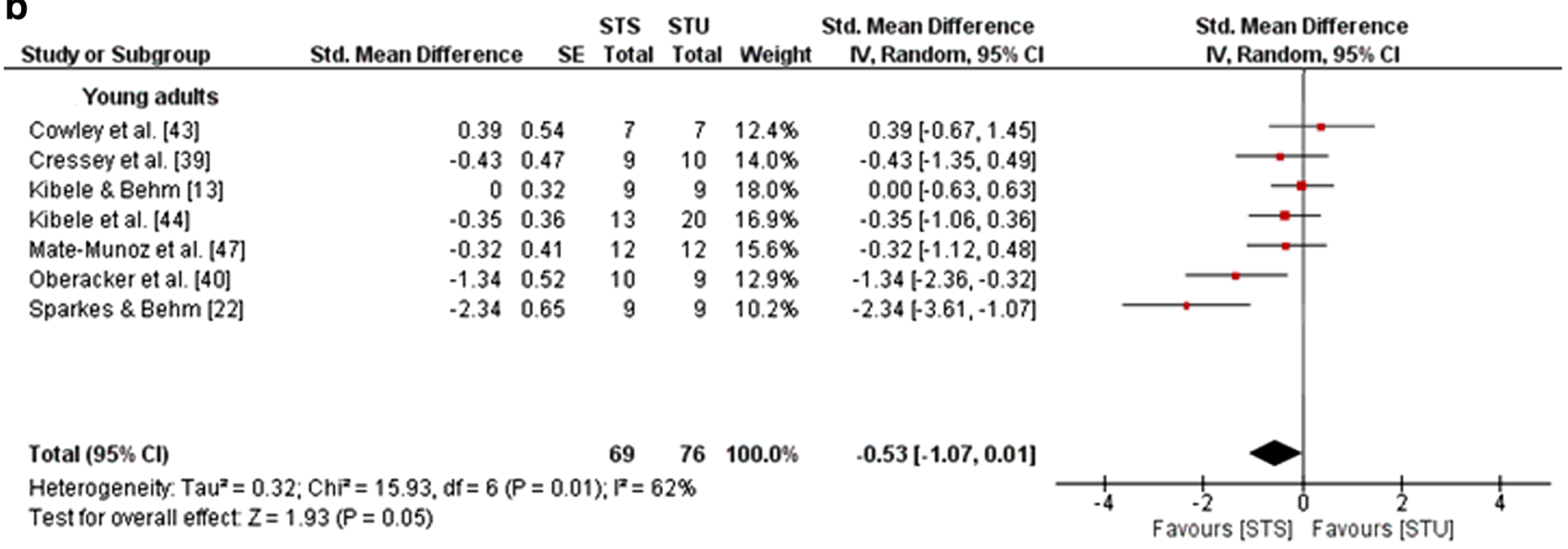

Fig. 7 Effects of strength training on unstable surfaces (STU) versus stable surfaces (STS) on measures of muscle power in healthy adolescents (a) and young adults (b). $C I$ confidence interval, $d f$ degrees of freedom, $I V$ inverse variance, $S E$ standard error, $S t d$. standard

trained athletes" (p. 110). However, the present results suggest that at least for non-elite athletes, there is a strength and power training stress/intensity plateau that is sufficient to induce positive training adaptations. In their review, Behm and Colado [8] reported that the mean force deficit with STU compared with similar STS exercises was $29 \%$. The present review indicates that in comparison with STU, exceeding that plateau by introducing greater strength or power challenges with STS does not provide significant advantages across the lifespan. As there were no agespecific differences in the training response, STU can be employed by the non-elite training population to improve strength and power and to achieve functional health benefits. In fact, the approximate STU-induced $30 \%$ force deficit [8] could be viewed alternatively as a benefit, as the lower external forces or torques might decrease the chance or incidence of training-related injuries or might be more beneficial for rehabilitation of an injured muscle group [8].

Furthermore, the American College of Sports Medicine recommends that older adults should conduct strength training using light loads (40-50\% 1RM) at the beginning of training and moderate loads near the end of training (60-70 \% 1RM) [55]. Similarly, low- to moderate-intensity strength training is recommended in youth [17]. Hence, the instability-related lower force outputs during performance of STU may not represent a compromising issue regarding neuromuscular adaptive processes in old adults and youth. Further, there is evidence in the literature that even in young healthy adults, strength training using low compared with high loads is equally effective in enhancing muscle strength. For example, isometric strength training at 100 versus $60 \%$ of maximal voluntary contraction (MVC) [56] or dynamic strength training at 55-60 versus $80-90 \%$ $1 \mathrm{RM}$ [57] resulted in similar improvements in measures of muscle strength. Thus, there is evidence that application of lower loads during STU provides a sufficient training stimulus to ensure similar strength or power training gains in comparison with STS using higher loads in different age groups. Of note, the study methodology of the included studies has to be taken into account when interpreting our findings. In other words, the included training studies ranged from 2 to 12 weeks and were conducted in primarily untrained or recreationally active individuals.

A potential reason for the observed similar training-induced adaptations following STU compared with STS could be related to similar or even higher levels of muscle 


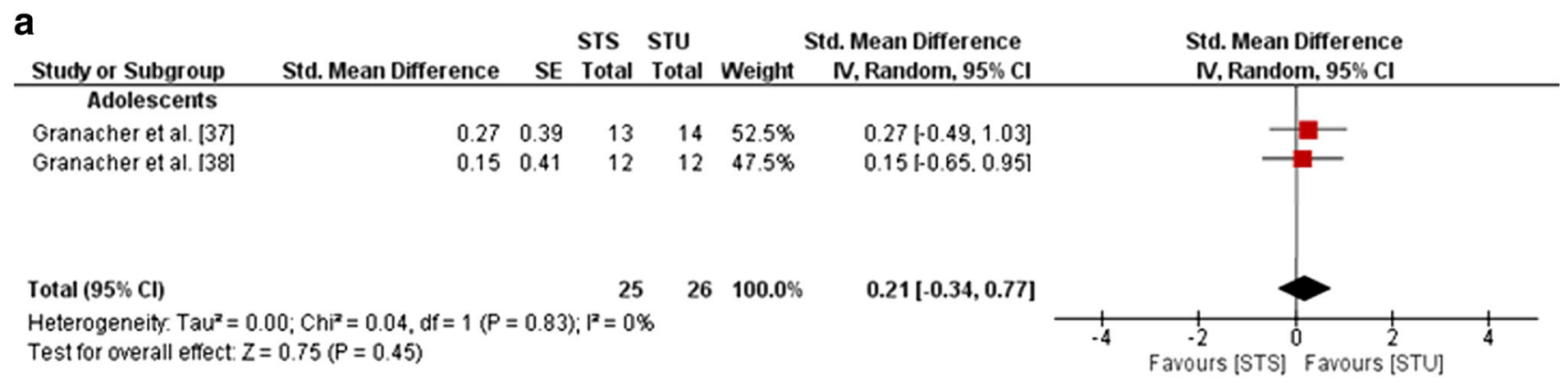

b

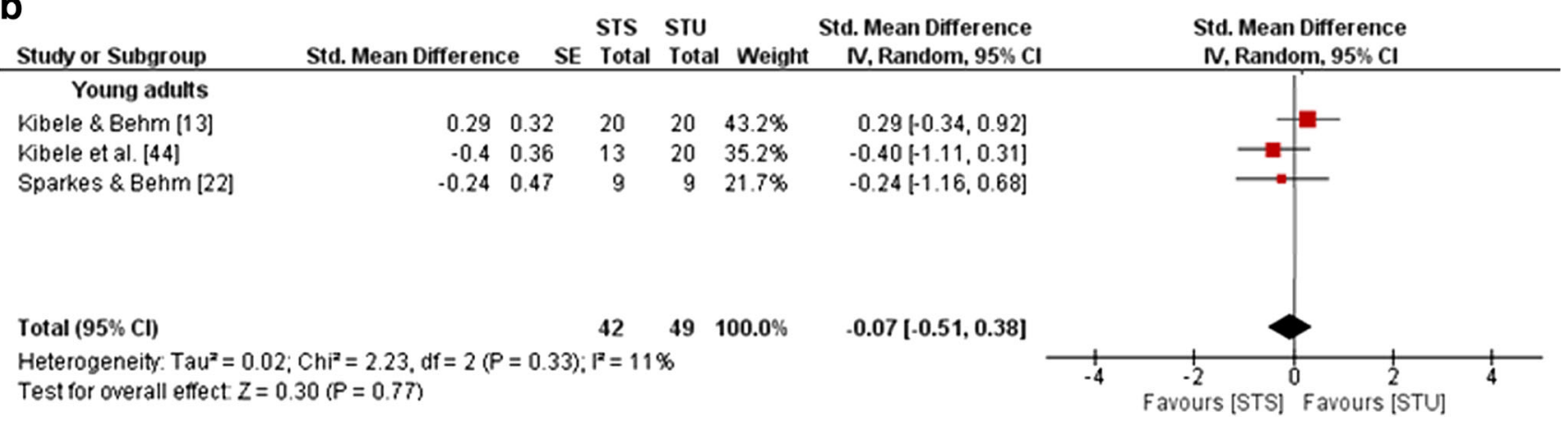

Fig. 8 Effects of strength training on unstable surfaces (STU) versus stable surfaces (STS) on measures of static balance in healthy adolescents (a) and young adults (b). $C I$ confidence interval, $d f$ degrees of freedom, $I V$ inverse variance, $S E$ standard error, $S t d$. standard

activation during performance of STU [49, 58]. In fact, Anderson and Behm [49] found no significant differences in overall electromyographic (EMG) activity of trunk and shoulder muscles during performance of a chest press exercise on an unstable surface (i.e. a Swiss ball) as compared with a stable surface (i.e. a bench). Further, significantly higher trunk muscle activity was observed during performance of squat movements on an unstable surface (i.e. performed with a balance disc under each foot) versus a stable surface (i.e. performed with a Smith machine) [58].

Our findings did not support our initial hypothesis regarding the greater effectiveness of STU in youth compared with adults. When comparing STU with CON, our results revealed medium effects of STU on measures of maximal strength in young adults and, depending on the analysed parameter, no effects (i.e. maximal strength of the trunk rotators right) to medium effects [i.e. maximal strength of the trunk flexors, rotators, lateral flexors (left/ right), lateral rotators (left)] in old adults. On the basis of these results, it can be concluded that STU provides an adequate stimulus to increase maximal strength in seniors, which is mostly equal to that observed in young adults. Further, comparison of STU and STS revealed similar training-induced performance enhancements for muscle endurance and dynamic balance in both adolescents and young adults. Methodological reasons may account for this somewhat unexpected finding. However, in terms of training, similar core strength, as well as plyometric exercises, on unstable surfaces/devices were included in adolescent [36-38] and adult [23, 31] training protocols. Further, in terms of testing, differences in the sensitivity of the applied strength, power and balance tests may also have been responsible for the unexpected findings. Yet, similar test modalities (e.g. CMJ, one-legged stance), equipment (e.g. force plate) and parameters (e.g. jump height, postural sway) were used in studies that investigated adolescents $[36,38]$ and young adults [44, 47]. Therefore, methodological reasons appear not to be responsible for our findings. This is why we suggest that instability-related reductions in absolute training loads during STU as compared with STS may explain our results because the reduced loads are not challenging enough to induce extra adaptive processes in the adolescent neuromuscular system. In fact, adolescence is characterized by significant increases in levels of circulating androgens (e.g. testosterone), particularly in boys $[59,60]$, which is why high training loads appear to be more suitable to induce marked increases in muscle mass and strength in this age group. As a limitation of this study, it has to be noted that only four studies were found that investigated the impact of STU versus STS or CON in adolescents and only three studies were found that investigated the impact of STU versus STS or CON in old adults. On the basis of the rather small 


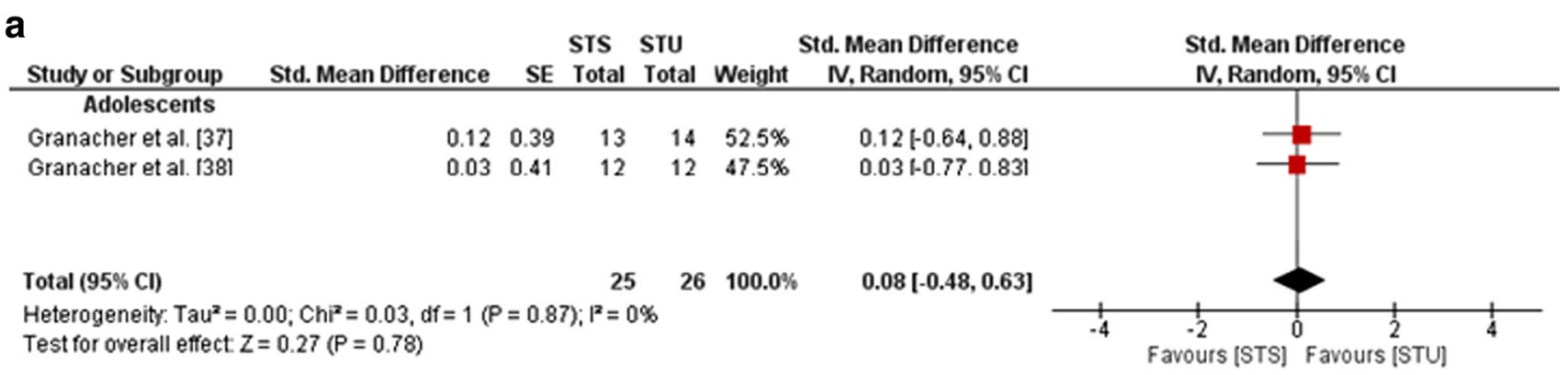

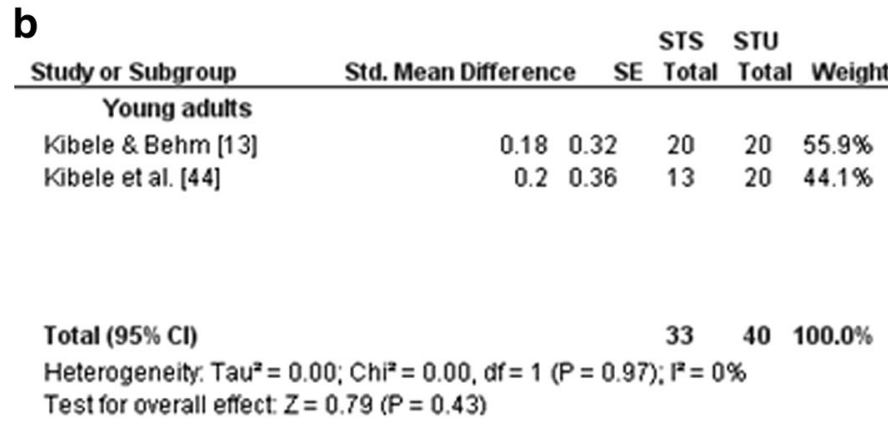

Std. Mean Difference
N. Random, 95\% Cl

Fig. 9 Effects of strength training on unstable surfaces (STU) versus stable surfaces (STS) on measures of dynamic balance in healthy adolescents (a) and young adults (b). $C I$ confidence interval, $d f$ degrees of freedom, $I V$ inverse variance, $S E$ standard error, Std. standard

number of studies, we consider our findings as preliminary. Therefore, further research is needed to determine the general effectiveness of STU as compared with no training or regular training. Equally or even more important is the need to elucidate the specific effects of STU as compared with other strength training programs (e.g. STS using high/ low loads).

\section{Conclusions}

This systematic review and meta-analysis shows that STU, when compared with no training or regular training only, is effective in improving strength performance in adolescents, young adults and old adults, as well as power and balance performance in young and old adults. However, heterogeneous effects were particularly found in adolescents and young adults when the effects of STU were compared with those of STS. Therefore, we conclude that the application of STU compared with STS has limited additional effects on measures of muscle strength, power and balance in healthy adolescents and young adults. Therefore, the use of unstable as compared with stable surfaces during strength training is only partially recommended. Because our systematic literature search did not identify studies that investigated the effects of STU versus STS in children, middle-aged adults and old adults, further research of high methodological quality (i.e. randomized controlled trials) is needed to determine whether there are extra effects of STU on muscle strength, power and balance performances in those age groups.

\section{Compliance with Ethical Standards}

This work was supported by a grant from the German Research Foundation (no. MU 3327/2-1). David G. Behm, Thomas Muehlbauer, Armin Kibele and Urs Granacher declare that they have no conflicts of interest.

Open Access This article is distributed under the terms of the Creative Commons Attribution 4.0 International License (http:// creativecommons.org/licenses/by/4.0/), which permits unrestricted use, distribution, and reproduction in any medium, provided you give appropriate credit to the original author(s) and the source, provide a link to the Creative Commons license, and indicate if changes were made.

\section{References}

1. Behm DG, Drinkwater EJ, Willardson JM, et al. The use of instability to train the core musculature. Appl Physiol Nutr Metab. 2010;35(1):91-108.

2. Behm DG, Colado Sanchez JC. Instability resistance training across the exercise continuum. Sports Health. 2013;5(6):500-3.

3. Behm DG. Neuromuscular implications and applications of resistance training. J Strength Cond Res. 1995;9(4):264-74.

4. Behm DG, Sale DG. Velocity specificity of resistance training. Sports Med. 1993;15(6):374-88.

5. Willardson JM. The effectiveness of resistance exercises performed on unstable equipment. Strength Cond J. 2004;26(5):70-4. 
6. Schmidtbleicher D. Training for power events. In: Komi PV, editor. Strength and power in sport. Oxford: Blackwell; 2004. p. 381-95.

7. Behm DG, Drinkwater EJ, Willardson JM, et al. Canadian Society for Exercise Physiology position stand: the use of instability to train the core in athletic and nonathletic conditioning. Appl Physiol Nutr Metab. 2010;35(1):109-12.

8. Behm D, Colado JC. The effectiveness of resistance training using unstable surfaces and devices for rehabilitation. Int J Sports Phys Ther. 2012;7(2):226-41.

9. Behm DG, Drinkwater EJ, Willardson JM, et al. The role of instability rehabilitative resistance training for core musculature. Strength Cond J. 2011;33(3):72-81.

10. Hamlyn N, Behm DG, Young WB. Trunk muscle activation during dynamic weight-training exercises and isometric instability activities. J Strength Cond Res. 2007;21(4):1108-12.

11. Chek P. Physioball exercise for swimming, soccer, and basketball. Sports Coach. 1999;21:30-3.

12. Verstegen M, Williams P. Physioball routine. In: Verstegen M, Williams P, editors. Core performance. New York: Rodale; 2004. p. $73-88$.

13. Kibele A, Behm DG. Seven weeks of instability and traditional resistance training effects on strength, balance and functional performance. J Strength Cond Res. 2009;23(9):2443-50.

14. Viru A, Loko J, Harro M, et al. Critical periods in the development of performance capacity during childhood and adolescence. Eur J Phys Edu. 1999;4(1):75-119.

15. Landow L, Haff GG. Use of stability balls in strength and conditioning. Strength Cond J. 2012;34(1):48-50.

16. Sackett DL, Straus SE, Richardson WS, et al. Evidence-based medicine: how to practice and teach EBM. Edinburgh: Churchill Livingstone; 2000.

17. Faigenbaum AD, Westcott WL, Loud RL, et al. The effects of different resistance training protocols on muscular strength and endurance development in children. Pediatrics. 1999;104(1):e5.

18. Granacher U, Lacroix A, Muehlbauer T, et al. Effects of core instability strength training on trunk muscle strength, spinal mobility, dynamic balance and functional mobility in older adults. Gerontology. 2013;59(2):105-13.

19. Moher D, Liberati A, Tetzlaff J, et al. Preferred reporting items for systematic reviews and meta-analyses: the PRISMA statement. J Clin Epidemiol. 2009;62(10):1006-12.

20. Anderson K, Behm DG. The impact of instability resistance training on balance and stability. Sports Med. 2005;35(1):43-53.

21. Behm DG, Anderson KG. The role of instability with resistance training. J Strength Cond Res. 2006;20(3):716-22.

22. Sparkes R, Behm DG. Training adaptations associated with an 8-week instability resistance training program with recreationally active individuals. J Strength Cond Res. 2010;24(7):1931-41.

23. Sukalinggam CL, Sukalinggam GL, Kasim F, et al. Stability ball training on lower back strength has greater effect in untrained female compared to male. J Hum Kinet. 2012;33:133-41.

24. Cosio-Lima LM, Reynolds KL, Winter C, et al. Effects of physioball and conventional floor exercises on early phase adaptations in back and abdominal core stability and balance in women. J Strength Cond Res. 2003;17(4):721-5.

25. Romero-Franco N, Martinez-Lopez E, Lomas-Vega R, et al. Effects of proprioceptive training program on core stability and center of gravity control in sprinters. J Strength Cond Res. 2012;26(8):2071-7.

26. Maher CG, Sherrington C, Herbert RD, et al. Reliability of the PEDro Scale for rating quality of randomized controlled trials. Phys Ther. 2003;83(8):713-21.

27. Deeks JJ, Higgins JPT. Statistical algorithms in Review Manager 5. The Cochrane Collaboration. 2010. Available from URL:
http://tech.cochrane.org/revman/documentation/Statistical-methods -in-RevMan-5.pdf. Accessed 29 Aug 2015.

28. Hedges LV, Olkin I. Statistical methods for meta-analysis. Orlando: Academic Press; 1985.

29. Cohen J. Statistical power for the behavioral sciences. Hillsdale: Erlbaum; 1988.

30. Deeks JJ, Higgins JPT, Altman DG. Chapter 9: analysing data and undertaking meta-analyses. In: Higgins JPT, Green S, editors. Cochrane handbook for systematic reviews of interventions. Bognor Regis: Wiley-Blackwell; 2008.

31. Stanforth D, Stanforth PR, Hahn SR, et al. A 10-week training study comparing Resistaball and traditional trunk training. J Dance Med Sci. 1998;2(4):134-40.

32. Marinkovic M, Bratic M, Ignjatovic A, et al. Effects of 8-week instability resistance training on maximal strength in inexperienced young individuals. Serb J Sports Sci. 2012;6(1):17-21.

33. Premkumar M, Alagesan J, Vaidya N. Effect of instability resistance training of abdominal muscles in healthy young females-an experimental study. Int J Pharm Sci Health Care. 2012;2(3):91-7.

34. Seo B-D, Yun Y-D, Kim H-R, et al. Effect of 12-week swiss ball exercise program on physical fitness and balance ability of elderly women. J Phys Ther Sci. 2012;24(1):11-5.

35. Stanton R, Reaburn PR, Humphries B. The effect of short-term Swiss ball training on core stability and running economy. J Strength Cond Res. 2004;18(3):522-8.

36. Prieske O, Muehlbauer T, Borde R, et al. Neuromuscular and athletic performance following core strength training in elite youth soccer: role of instability. Scand J Med Sci Sports. 2015. doi:10.1111/sms.12403 (Epub 2015 Jan 6).

37. Granacher U, Schellbach J, Klein K, et al. Effects of core strength training using stable versus unstable surfaces on physical fitness in adolescents: a randomized controlled trial. BMC Sports Sci Med Rehabil. 2014;6:40.

38. Granacher U, Prieske O, Majewski M, et al. The role of instability with plyometric training in sub-elite adolescent soccer players. Int J Sports Med. 2015;36(5):386-94.

39. Cressey EM, West CA, Tiberio DP, et al. The effects of ten weeks of lower-body unstable surface training on markers of athletic performance. J Strength Cond Res. 2007;21(2):561-7.

40. Oberacker LM, Davis SE, Haff GG, et al. The Yo-Yo IR2 test: physiological response, reliability, and application to elite soccer. J Strength Cond Res. 2012;26(10):2734-40.

41. Sato K, Mokha M. Does core strength training influence running kinetics, lower-extremity stability, and 5000-M performance in runners? J Strength Cond Res. 2009;23(1):133-40.

42. Carter JM, Beam WC, McMahan SG, et al. The effects of stability ball training on spinal stability in sedentary individuals. J Strength Cond Res. 2006;20(2):429-35.

43. Cowley PM, Swensen T, Sforzo GA. Efficacy of instability resistance training. Int J Sports Med. 2007;28(10):829-35.

44. Kibele A, Classen C, Muehlbauer T, et al. Metastability in plyometric training on unstable surfaces: a pilot study. BMC Sports Sci Med Rehabil. 2014;6:30.

45. Chulvi-Medrano I, Martinez-Ballester E, Masia-Tortosa L. Comparison of the effects of an eight-week push-up program using stable versus unstable surfaces. Int J Sports Phys Ther. 2012;7(6):586-94.

46. Cug M, Ak E, Özdemir RA, et al. The effect of instability training on knee joint proprioception and core strength. J Sports Sci Med. 2012;11(3):468-74.

47. Mate-Munoz JL, Monroy AJ, Jodra Jimenez P, et al. Effects of instability versus traditional resistance training on strength, power and velocity in untrained men. J Sports Sci Med. 2014;13(3):460-8. 
48. Chulvi-Medrano I, Colado JC, Pablos C, et al. A lower-limb training program to improve balance in healthy elderly women using the T-bow device. Phys Sportsmed. 2009;37(2):127-35.

49. Anderson KG, Behm DG. Maintenance of EMG activity and loss of force output with instability. J Strength Cond Res. 2004;18(3):637-40.

50. Drinkwater EJ, Pritchett EJ, Behm DG. Effect of instability and resistance on unintentional squat-lifting kinetics. Int J Sports Physiol Perform. 2007;2(4):400-13.

51. Saeterbakken AH, Fimland MS. Muscle force output and electromyographic activity in squats with various unstable surfaces. J Strength Cond Res. 2013;27(1):130-6.

52. Saeterbakken AH, Fimland MS. Electromyographic activity and 6RM strength in bench press on stable and unstable surfaces. J Strength Cond Res. 2013;27(4):1101-7.

53. Goodman CA, Pearce AJ, Nicholes CJ, et al. No difference in 1 RM strength and muscle activation during the barbell chest press on a stable and unstable surface. J Strength Cond Res. 2008;22(1):88-94.

54. Koshida S, Urabe Y, Miyashita K, et al. Muscular outputs during dynamic bench press under stable versus unstable conditions. J Strength Cond Res. 2008;22(5):1584-8.
55. ACSM. ACSM's guidelines for exercise testing and prescription. 9th ed. Philadelphia: Wolters Kluwer; 2013.

56. Kanehisa H, Nagareda H, Kawakami Y, et al. Effects of equivolume isometric training programs comprising medium or high resistance on muscle size and strength. Eur J Appl Physiol. 2002;87:112-9.

57. Tanimoto M, Sanada K, Yamamoto K, et al. Effects of wholebody low-intensity resistance training with slow movement and tonic force generation on muscular size and strength in young men. J Strength Cond Res. 2008;22(6):1926-38.

58. Anderson K, Behm DG. Trunk muscle activity increases with unstable squat movements. Can J Appl Physiol. 2005;30(1):33-45.

59. Blimkie CJ. Age- and sex-associated variation in strength during childhood: anthropometric, morphologic, neurologic, biomechanical, endocrinologic, genetic, and physical activity correlates. In: Gisolfi CV, Lamb DR, editors. Perspectives in exercise science and sports medicine: youth, exercise, and sport. Indianapolis: Benchmark; 1989. p. 99-163.

60. Round JM, Jones DA, Honour JW, et al. Hormonal factors in the development of differences in strength between boys and girls during adolescence: a longitudinal study. Ann Hum Biol. 1999;26(1):49-62. 